\title{
Spinning Witten diagrams
}

\section{Charlotte Sleight and Massimo Taronna ${ }^{1}$}

Université Libre de Bruxelles and International Solvay Institutes, ULB-Campus Plaine CP231, 1050 Brussels, Belgium

E-mail: charlotte.sleight@gmail.com, massimo.taronna@ulb.ac.be

ABSTRACT: We develop a systematic framework to compute the conformal partial wave expansions (CPWEs) of tree-level four-point Witten diagrams with totally symmetric external fields of arbitrary mass and integer spin in $\mathrm{AdS}_{d+1}$. As an intermediate step, we identify convenient bases of three-point bulk and boundary structures to invert linear map between spinning three-point conformal structures and spinning cubic couplings in AdS. Given a $\mathrm{CFT}_{d}$, this provides the complete holographic reconstruction of all cubic couplings involving totally symmetric fields in the putative dual theory on $\mathrm{AdS}_{d+1}$. Employing this framework, we determine the CPWE of a generic four-point exchange Witten diagram with spinning exchanged field. As a concrete application, we compute all four-point exchange Witten diagrams in the type A higher-spin gauge theory on $\mathrm{AdS}_{d+1}$, which is conjectured to be dual to the free scalar $O(N)$ model.

Keywords: AdS-CFT Correspondence, Conformal Field Theory, Higher Spin Gravity, Higher Spin Symmetry

ArXiv EPrint: 1702.08619

\footnotetext{
${ }^{1}$ Postdoctoral Researcher of the Fund for Scientific Research-FNRS Belgium.
} 


\section{Contents}

1 Introduction 1

2 Conformal partial waves 3

2.1 The conformal partial wave expansion 3

2.2 Spinning conformal partial waves 4

2.2.1 External scalar operators 5

2.2.2 Spinning conformal partial waves 6

$\begin{array}{lll}2.2 .3 & \text { Spinning conserved conformal partial waves } & 7\end{array}$

3 CPWE of spinning Witten diagrams $\quad 9$

$\begin{array}{ll}3.1 & \text { Spinning three-point Witten diagrams } \\ \end{array}$

$\begin{array}{lll}3.1 .1 & \text { Building blocks of cubic vertices } & 11\end{array}$

3.1.2 Spinning Witten diagrams from a scalar seed 12

$\begin{array}{ll}\text { 3.1.3 A natural basis of cubic structures in AdS/CFT } & 14\end{array}$

$\begin{array}{ll}3.2 & \text { Spinning bulk-to-bulk propagators } \\ & 16\end{array}$

$\begin{array}{lll}3.2 .1 & \text { Massive case } & 17\end{array}$

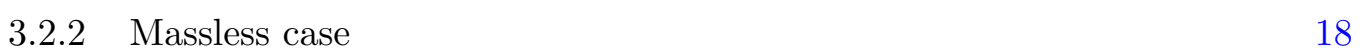

3.3 CPWE of spinning exchange diagrams 20

3.3.1 Natural basis of conformal partial waves in AdS/CFT 20

$\begin{array}{ll}\text { 3.3.2 Generic spinning exchange diagram } & 21\end{array}$

3.4 Spinning exchanges in the type A higher-spin gauge theory 24

$\begin{array}{lll}3.4 .1 & \text { Off-shell cubic couplings } & 24\end{array}$

$\begin{array}{lll}3.4 .2 & \text { Four-point exchange diagrams } & 25\end{array}$

$\begin{array}{lr}\text { A Conventions, notations and ambient space } & 28\end{array}$

$\begin{array}{ll}\text { B The improved current } & 30\end{array}$

$\begin{array}{ll}\text { C Trace of the currents } & 31\end{array}$

D Seed bulk integrals $\quad 32$

\section{Introduction}

Conformal field theories (CFT) are among the most well studied examples of quantum field theories (QFT), and are also among the few which admit a simple non-perturbative definition. This is owing to the fact that conformal invariance fixes all $2 \mathrm{pt}$ and $3 \mathrm{pt}$ correlation functions up to numerical coefficients and spectrum, usually referred to as CFT 
data. Associativity of the conformal operator algebra then allows to reconstruct, in principle, all higher point correlation functions at the non-perturbative level. This intrinsic simplicity triggered the pioneering works [1-6] centred on the idea that symmetry and quantum mechanics alone should suffice to fix the dynamics of a QFT. This is known as the Bootstrap Program. This approach proved to be very successful in the 80's in the context of 2d CFTs [7], but remained dormant for CFTs in $d>2$ until very recently with the emergence of new analytic and numerical methods [8-15]. These have led to striking new numerical results for 3d CFTs [16, 17], and have further triggered new analytic results for the conformal bootstrap in various limits [18-23].

CFTs also play a pivotal role in the holographic dualities, and are conjectured to be dual to gravitational theories living in a higher-dimensional anti-de Sitter (AdS) space [2426]. From a bottom up perspective, AdS/CFT maps bulk and boundary consistency into each other, repackaging the various kinematic building blocks in terms of bulk or boundary degrees of freedom. To some extent, without imposing any additional constraint, this is a kinematic re-writing of the same physical object in two different bases. It was further shown in $[27,28]$ that, in the large $N$ limit, standard Feynman diagram expansion in the bulk does repackage solutions to the bootstrap at leading order in $\frac{1}{N}$. In particular, this repackaging is in terms of Witten diagrams. From the bulk perspective, the latter play the role of the building blocks in terms of which the observables of the theory are expressed - in direct analogy with S-matrix elements. In this holographic picture, a crucial physical consistency requirement for a non-trivial field theory description in the bulk is that of locality, see e.g. [27, 29-38] for an incomplete list of works in this direction. ${ }^{1}$

Holography thus naturally provides a reformulation of the bootstrap problem in terms of different types of building blocks, which have a neat physical interpretation. The link between these two pictures is the main subject of the present work. In particular, we explicitly invert the map between spinning three-point conformal structures and CPWE expansion on the boundary, and the local spinning bulk cubic couplings and Witten diagrams in the bulk. At the level of four-point functions this draws upon the link [37, 40] between the shadow formalism $[1,4,41,42]$ and the split representation of AdS harmonic functions [43]. At the level of three-point functions, given a $\mathrm{CFT}_{d}$ our results provide the complete holographic reconstruction of all cubic couplings involving totally symmetric fields in the putative dual theory on $\mathrm{AdS}_{d+1}$. Previous works on the holographic reconstruction of bulk interactions from CFT correlation functions include: [31, 37, 44-47] in the context of higher-spin holography, and more recently in the context of $p$-adic AdS/CFT [49].

A key motivation behind this work is that such a bulk repackaging of CFT objects may give new insights into the bootstrap program, potentially providing novel methods to solve the crossing equations (see [22, 50-52] for recent progress in this direction). Furthermore, this may also shed light on the quest for understanding quantum gravity and which CFTs admit a well-defined gravitational dual.

\footnotetext{
${ }^{1}$ The main issue is that, with no restriction on the functional class of interactions, a field theory description in the bulk becomes a tautological re-writing of CFT data (see e.g. [39] for a theorem on the triviality of interactions in the absence of any locality requirement.)
} 
In the process of diagonalising the map between boundary OPE coefficients and bulk cubic couplings, we identify the corresponding bases of bulk and boundary $3 \mathrm{pt}$ and $4 \mathrm{pt}$ structures, which, in this sense, appear to be naturally selected by holography. This allows us to systematically study tree-level four-point exchange amplitudes involving totally symmetric fields of arbitrary mass and spin, and seamlessly derive their CPWE. Our formalism builds upon, and extends, the approach and results of the previous works [31, 37, 40, 45], which considered four-point Witten diagrams with only external scalars. As a concrete application, we determine all four-point exchange diagrams in the type-A higher-spin gauge theory on $\mathrm{AdS}_{d+1}$, whose complete cubic couplings have been recently been established in metric-like form in $[47,53]$. This application of our results extends the previous exchange diagram computations [31, 45] in the type A higher-spin gauge theory to include external gauge fields of arbitrary integer spin.

Let us also mention a parallel approach to the conformal partial wave decomposition of Witten diagrams, which has recently been developed in [54]. ${ }^{2}$ This is underpinned by what is known as the "geodesic Witten diagram", the bulk object which computes a single conformal partial wave. The latter is essentially an exchange Witten diagram, but the crucial difference being that one integrates the cubic vertices over geodesics, as opposed to the full volume of AdS. The original paper [54] considered the case of external scalars, which has more recently been generalised to external fields with arbitrary integer spin: first to a single spinning external leg in [63], and very recently to each leg having arbitrary integer spin in [64-66]. It would be instructive to employ the geodesic Witten diagram approach developed in [54] to reproduce the explicit results obtained in section 3.3 for the CPWEs of spinning exchange Witten diagrams. A prescription for the latter very recently appeared in [64], together with some results for spin-0 and spin-1 exchange diagrams.

The outline is as follows: section 2 we review the CPWE in the standard setting of CFT, with a particular focus on the shadow formalism. In section 3 we detail the parallel story in the bulk. In particular, how the harmonic function decomposition of four-point Witten diagrams provides the link with the shadow formalism via the split representation. In section 3.1 we review the computation [47] of generic spinning three point Witten diagrams, and present a convenient explicit diagonal form of the linear map between three-point conformal structures and local bulk cubic couplings. In section 3.3 we apply the latter results to compute the CPWE of a generic spinning exchange Witten diagram in $\mathrm{AdS}_{d+1}$, and furthermore in section 3.4 consider exchange diagrams in the concrete setting of the type A minimal higher-spin gauge theory. Various technical details are relegated to appendices $\mathrm{A}, \mathrm{B}, \mathrm{C}$ and $\mathrm{D}$.

\section{Conformal partial waves}

\subsection{The conformal partial wave expansion}

The CPWE of correlation functions of primary operators in CFT is a decomposition into contributions from each conformal multiplet. ${ }^{3}$ As a simple illustrative example, let us first

\footnotetext{
${ }^{2}$ This has origins in the $\mathrm{AdS}_{3} / \mathrm{CFT}_{2}$ literature [55-62], on Virasoro and $\mathcal{W}_{N}$ conformal partial waves from the bulk.

${ }^{3}$ I.e. each conformal partial wave re-sums the contribution of the primary operator + all of its descendants to the correlator, and is thus labelled by the dimension $\Delta$ and spin $s$ of the primary operator.
} 
consider the CPWEs of correlation functions involving scalar primary operators $\mathcal{O}_{i}$. For a four-point function expanded in the s-channel, ${ }^{4}$ this reads

$$
\left\langle\mathcal{O}_{1}\left(y_{1}\right) \mathcal{O}_{2}\left(y_{2}\right) \mathcal{O}_{3}\left(y_{3}\right) \mathcal{O}_{4}\left(y_{4}\right)\right\rangle=\sum_{\mathcal{O}_{\Delta, s}} \boldsymbol{c}_{\mathcal{O}_{1} \mathcal{O}_{2} \mathcal{O}_{\Delta, s}} c^{\mathcal{O}_{\Delta, s}} \mathcal{O}_{3} \mathcal{O}_{4} W_{\Delta, s}\left(y_{i}\right) .
$$

The functions $W_{\Delta, s}$ are the conformal partial waves. These are purely kinematical objects, fixed completely by conformal symmetry and only depend on the representations of the primary operators $\mathcal{O}_{\Delta, s}$ and $\mathcal{O}_{i}$ under the conformal group. Each conformal partial wave in the expansion (2.1) is weighted by the coefficients of the operator $\mathcal{O}_{\Delta, s}$ in the $\mathcal{O}_{1} \times \mathcal{O}_{2}$ and $\mathcal{O}_{3} \times \mathcal{O}_{4}$ OPEs. The CPWE thus effectively disentangles the dynamical information, which depends on the theory under consideration, from the universal information dictated by conformal symmetry.

Owing to these defining features, the CPWE expansion has turned out to be a powerful tool. This is highlighted, for instance, by its pivotal role in the successes $([10,11,16,67,68]$, to name a few) of the conformal bootstrap program $[3,5]$. But in spite of this, explicit closed formulas for conformal partial waves are only possible in certain cases. For the scalar case (2.1) closed form expressions are only available in even dimensions [8,9], while in other cases CPWs are inferred via indirect methods, such as: recursion relations [13, 69-73] and efficient series expansions [74-76].

In the following section we review another indirect approach, which is convenient for the CPWE of correlators involving operators with spin - as well as their Witten diagram counterparts. This is underpinned by the shadow formalism of Ferrara, Gatto, Grillo, and Parisi [1, 4, 41, 42], and leads to an expression for conformal blocks for operators in arbitrary Lorentz representations as an integral of three-point conformal structures. This approach was first considered by Hoffmann, Petkou and Rühl in [77, 78] for external scalar operators (see also [13]), and the idea revisited and results generalised in [79-81].

\subsection{Spinning conformal partial waves}

To a given primary operator $\mathcal{O}_{\Delta, s}$, can be associated a dual (or shadow) operator ${ }^{5}$

$$
\tilde{\mathcal{O}}_{\Delta, s}(y ; z)=\kappa_{\Delta, s} \frac{1}{\pi^{d / 2}} \int d^{d} y^{\prime} \frac{1}{\left(y-y^{\prime}\right)^{2 d-2 \Delta}}\left(z \cdot I\left(y-y^{\prime}\right) \cdot \hat{\partial}_{z^{\prime}}\right)^{s} \mathcal{O}_{\Delta, s}\left(y^{\prime} ; z^{\prime}\right),
$$

of the same spin and scaling dimension $d-\Delta$. The normalisation

$$
\kappa_{\Delta, s}=\frac{\Gamma(d-\Delta+s)}{\Gamma\left(\Delta-\frac{d}{2}\right)} \frac{1}{(\Delta-1)_{s}}
$$

ensures that applying (2.4) twice gives the identity.

\footnotetext{
${ }^{4}$ We use sans-serif font to denote the expansion channels, to be distinguished from the spin, $s$.

${ }^{5} I_{\mu \nu}(y)$ is the inversion tensor

$$
I_{\mu \nu}(y)=\delta_{\mu \nu}-\frac{2 y_{\mu} y_{\nu}}{y^{2}} ; \quad z_{1} \cdot I(y) \cdot z_{2}=z_{1} \cdot z_{2}-2 \frac{z_{1} \cdot y z_{2} \cdot y}{y^{2}} .
$$
}

The Thomas derivative [82] (see also [83])

$$
\hat{\partial}_{z^{i}}=\partial_{z^{i}}-\frac{1}{d-2+2 z \cdot \partial_{z}} z_{i} \partial_{z}^{2},
$$

accounts for tracelessness, i.e. $z^{2}=0$. 
The key observation of the shadow approach to conformal partial waves is that the integral

$$
\mathcal{P}_{\Delta, s}=\kappa_{d-\Delta, s} \frac{1}{\pi^{d / 2}} \int d^{d} y \mathcal{O}_{\Delta, s}(y)|0\rangle\langle 0| \tilde{\mathcal{O}}_{\Delta, s}(y),
$$

projects onto the contribution of the conformal families of $\mathcal{O}_{\Delta, s}$ and its shadow to a given four-point function. This is illustrated for the simplest case of scalar correlators in the following, before moving on to correlators of spinning operators.

\subsubsection{External scalar operators}

Restricting, for now, to the case of external scalar operators (2.1), when projecting onto the s-channel we have

$$
\begin{aligned}
\left\langle\mathcal{O}_{1}\left(y_{1}\right) \mathcal{O}_{2}\left(y_{2}\right) \mathcal{P}_{\Delta, s} \mathcal{O}_{3}\left(y_{3}\right) \mathcal{O}_{4}\left(y_{4}\right)\right\rangle & \\
& =\mathrm{c}_{\mathcal{O}_{1} \mathcal{O}_{2} \mathcal{O}_{\Delta, s}}{ }^{\mathcal{O}_{\Delta, s}} \mathcal{O}_{3} \mathcal{O}_{4} W_{\Delta, s}\left(y_{i}\right)+\mathrm{c}_{\mathcal{O}_{1} \mathcal{O}_{2} \tilde{\mathcal{O}}_{\Delta, s}}{ }^{\mathrm{c}^{\tilde{\mathcal{O}}}}{ }_{\mathcal{O}_{3} \mathcal{O}_{4}} W_{d-\Delta, s}\left(y_{i}\right),
\end{aligned}
$$

which implies the following integral representation

$$
\begin{aligned}
& \mathrm{C}_{\mathcal{O}_{1} \mathcal{O}_{2} \mathcal{O}_{\Delta, s} c^{\mathcal{O}_{\Delta, s}} \mathcal{O}_{3} \mathcal{O}_{4}} W_{\Delta, s}\left(y_{i}\right)+\text { shadow } \\
& \quad=\kappa_{d-\Delta, s} \frac{1}{\pi^{d / 2}} \int d^{d} y\left\langle\mathcal{O}_{1}\left(y_{1}\right) \mathcal{O}_{2}\left(y_{2}\right) \mathcal{O}_{\Delta, s}(y)\right\rangle\left\langle\tilde{\mathcal{O}}_{\Delta, s}(y) \mathcal{O}_{3}\left(y_{3}\right) \mathcal{O}_{4}\left(y_{4}\right)\right\rangle,
\end{aligned}
$$

for the total contribution as a product of two three-point functions. Stripping off the dynamical data leaves a universal integral expression for the sum of a conformal partial wave and its shadow, dictated purely by conformal symmetry and the operator representations:

$$
\begin{aligned}
W_{\Delta, s}\left(y_{i}\right)+ & \text { shadow } \\
& =\kappa_{d-\Delta, s} \frac{\gamma_{\tau, s} \bar{\gamma}_{\tau, s}}{\pi^{d / 2}} \int d^{d} y\left\langle\left\langle\mathcal{O}_{1}\left(y_{1}\right) \mathcal{O}_{2}\left(y_{2}\right) \mathcal{O}_{\Delta, s}(y)\right\rangle\right\rangle\left\langle\left\langle\tilde{\mathcal{O}}_{\Delta, s}(y) \mathcal{O}_{3}\left(y_{3}\right) \mathcal{O}_{4}\left(y_{4}\right)\right\rangle\right\rangle
\end{aligned}
$$

where

$$
\gamma_{\tau, s}=\frac{\Gamma\left(\frac{d}{2}-\frac{\tau_{3}-\tau_{4}+\tau}{2}\right)}{\Gamma\left(\frac{\tau_{3}-\tau_{4}+\tau}{2}+s\right)}, \quad \bar{\gamma}_{\tau, s}=\frac{\Gamma\left(\frac{d}{2}-\frac{\tau_{4}-\tau_{3}+\tau}{2}\right)}{\Gamma\left(\frac{\tau_{4}-\tau_{3}+\tau}{2}+s\right)} .
$$

The notation $\langle\langle\bullet\rangle\rangle$ denotes the kinematical part of the three-point function that is fixed by conformal symmetry. I.e. removal of the overall coefficient, ${ }^{6}$

$$
\begin{aligned}
& \left\langle\mathcal{O}_{1}\left(y_{1}\right) \mathcal{O}_{2}\left(y_{2}\right) \mathcal{O}_{\Delta, s}(y)\right\rangle=\mathrm{c}_{\mathcal{O}_{1} \mathcal{O}_{2} \mathcal{O}_{\Delta, s}}\left\langle\left\langle\mathcal{O}_{1}\left(y_{1}\right) \mathcal{O}_{2}\left(y_{2}\right) \mathcal{O}_{\Delta, s}(y)\right\rangle\right\rangle \\
& \left\langle\tilde{\mathcal{O}}_{\Delta, s}(y) \mathcal{O}_{3}\left(y_{3}\right) \mathcal{O}_{4}\left(y_{4}\right)\right\rangle=\mathrm{c}_{\tilde{\mathcal{O}}_{\Delta, s} \mathcal{O}_{3} \mathcal{O}_{4}}\left\langle\left\langle\tilde{\mathcal{O}}_{\Delta, s}(y) \mathcal{O}_{3}\left(y_{3}\right) \mathcal{O}_{4}\left(y_{4}\right)\right\rangle\right\rangle,
\end{aligned}
$$

which, for unit two-point function normalisation, is the removal of the OPE coefficients. Details on the above steps where given by Dolan and Osborn in [13] section 3 and [8].

\footnotetext{
${ }^{6}$ Using the definition (2.4) one finds

$$
\mathrm{c}_{\tilde{\mathcal{O}}_{\Delta, s} \mathcal{O}_{3} \mathcal{O}_{4}}=\gamma_{\tau, s} \bar{\gamma}_{\tau, s} \mathrm{c}_{\mathcal{O}_{\Delta, s} \mathcal{O}_{3} \mathcal{O}_{4}},
$$
}

which is the origin of the factors (2.10) in the expression (2.9). 
An integral expression for a single, non-shadow, conformal partial wave can be obtained by introducing a contour integral ${ }^{7}$

$$
W_{\Delta, s}\left(y_{i}\right)=\frac{\left(\Delta-\frac{d}{2}\right)}{2 \pi} \int_{-\infty}^{\infty} \frac{d \nu}{\nu^{2}+\left(\Delta-\frac{d}{2}\right)^{2}}\left(W_{\frac{d}{2}+i \nu, s}\left(y_{i}\right)+W_{\frac{d}{2}-i \nu, s}\left(y_{i}\right)\right),
$$

and inserting (2.9) into the integrand. The CPWE (2.1) can then be re-cast as a contour integral [83, 84],

$$
\begin{aligned}
& \left\langle\mathcal{O}_{1}\left(y_{1}\right) \mathcal{O}_{2}\left(y_{2}\right) \mathcal{O}_{3}\left(y_{3}\right) \mathcal{O}_{4}\left(y_{4}\right)\right\rangle \\
& \quad=\sum_{s} \int_{-\infty}^{\infty} d \nu c_{s}(\nu) \int d^{d} y\left\langle\left\langle\mathcal{O}_{1}\left(y_{1}\right) \mathcal{O}_{2}\left(y_{2}\right) \mathcal{O}_{\frac{d}{2}+i \nu, s}(y)\right\rangle\right\rangle\left\langle\left\langle\mathcal{O}_{\frac{d}{2}-i \nu, s}(y) \mathcal{O}_{3}\left(y_{3}\right) \mathcal{O}_{4}\left(y_{4}\right)\right\rangle\right\rangle,
\end{aligned}
$$

where for ease of notation we defined $\mathcal{O}_{\frac{d}{2}-i \nu, s}=\tilde{\mathcal{O}}_{\frac{d}{2}+i \nu, s}$. The real function $c_{s}(\nu)$ encodes the dynamical information, with poles that carry the contribution from each spin- $s$ conformal multiplet. For example, a contribution from a conformal multiplet $[\Delta, s]$ manifests itself in $c_{s}(\nu)$ with a pole at $\frac{d}{2}+i \nu=\Delta$, with residue giving the OPE coefficients

$$
c_{s}(\nu)=\frac{\left(\Delta-\frac{d}{2}\right) \kappa_{d-\Delta, s} \gamma_{\tau, s} \bar{\gamma}_{\tau, s}}{2 \pi^{d / 2+1}} \frac{\mathrm{c}_{\mathcal{O}_{1} \mathcal{O}_{2} \mathcal{O}_{\Delta, s}{ }^{\mathcal{O}_{\Delta, s}} \mathcal{O}_{3} \mathcal{O}_{4}}}{\left(\frac{d}{2}-\Delta+i \nu\right)\left(\frac{d}{2}-\Delta-i \nu\right)}+\ldots,
$$

where the $\ldots$ denote possible contributions from other spin- $s$ multiplets in the spectrum. The contour integral form (2.14) of the CPWE admits a direct generalisation to fourpoint correlators involving operators with spin. The only difference with respect to the scalar case is that, in general, there is more than one conformal partial wave associated to each conformal multiplet. This is a consequence of the non-uniqueness of tensor structures compatible with conformal symmetry in three-point functions with more than one spinning operator. It is for this reason that external spinning operators are easily accommodated for in the integral form (2.13) of the conformal partial wave, which we discuss in the following.

\subsubsection{Spinning conformal partial waves}

The integral representation (2.13) of conformal partial waves carries over straightforwardly to CPWEs of four-point functions containing operators with spin. In this case, however, since the structure of three-point functions with more than one operator of non-zero spin is not unique, generally there is more than one conformal partial wave associated to the contribution of a given conformal multiplet.

The number of independent structures that may appear in a conformal three-point function with operators of spins $s_{1}-s_{2}-s_{3}$ is [85]

$$
N\left(s_{1}, s_{2}, s_{3}\right)=\frac{\left(s_{1}+1\right)\left(s_{1}+2\right)\left(3 s_{2}-s_{1}+3\right)}{6}-\frac{p(p+2)(2 p+5)}{24}-\frac{1-(-1)^{p}}{16},
$$

where $s_{1} \leq s_{2} \leq s_{3}$ and $p \equiv \operatorname{Max}\left(0, s_{1}+s_{2}-s_{3}\right)$. For correlation functions with two scalar operators there is just a single structure compatible with conformal symmetry,

\footnotetext{
${ }^{7}$ The conformal partial wave $W_{\frac{d}{2} \pm i \nu, s}\left(y_{i}\right)$ decays exponentially for $\operatorname{Im}(\nu) \rightarrow \mp \infty$. In applying the residue theorem to obtain the l.h.s. from the r.h.s., for $W_{\frac{d}{2} \pm i \nu, s}$ we close the $\nu$-contour in the lower/upper half plane respectively.
} 
$N(0,0, s)=1$, in accordance with the uniqueness of conformal partial waves with external scalar operators that we previously observed.

Three-point functions involving two spinning operators have $N\left(s_{1}, s_{2}, s_{3}\right)>1$. A general three-point function of spinning operators in a parity-even theory takes the form ${ }^{8}$

$$
\begin{aligned}
& \left\langle\mathcal{O}_{\Delta_{1}, s_{1}}\left(y_{1}\right) \mathcal{O}_{\Delta_{2}, s_{2}}\left(y_{2}\right) \mathcal{O}_{\Delta_{3}, s_{3}}\left(y_{3}\right)\right\rangle \\
& =\sum_{n_{i}} \mathrm{c}_{s_{1}, s_{2}, s_{3}}^{n_{1}, n_{2}, n_{3}} \frac{\mathrm{Y}_{1}^{s_{1}-n_{2}-n_{3}} \mathrm{Y}_{2}^{s_{2}-n_{3}-n_{1}} \mathrm{Y}_{3}^{s_{3}-n_{1}-n_{2}} \mathrm{H}_{1}^{n_{1}} \mathrm{H}_{2}^{n_{2}} \mathrm{H}_{3}^{n_{3}}}{\left(y_{12}^{2}\right)^{\frac{\tau_{1}+\tau_{2}-\tau_{3}}{2}}\left(y_{23}^{2}\right)^{\frac{\tau_{2}+\tau_{3}-\tau_{1}}{2}}\left(y_{31}^{2}\right)^{\frac{\tau_{3}+\tau_{1}-\tau_{2}}{2}}},
\end{aligned}
$$

with theory-dependent OPE coefficients $\mathrm{c}_{s_{1}, s_{2}, s_{3}}^{n_{1}, n_{2}, n_{3}}$. The six three-point conformally covariant building blocks are given by $(i \cong i+3)^{9}$

$$
\begin{aligned}
& \mathrm{Y}_{i}=\frac{z_{i} \cdot y_{i(i+1)}}{y_{i(i+1)}^{2}}-\frac{z_{i} \cdot y_{i(i+2)}}{y_{i(i+2)}^{2}}, \\
& \mathrm{H}_{i}=\frac{1}{y_{(i+1)(i+2)}^{2}}\left(z_{i+1} \cdot z_{i+2}+\frac{2 z_{i+1} \cdot y_{(i+1)(i+2)} z_{i+2} \cdot y_{(i+2)(i+1)}}{y_{(i+1)(i+2)}^{2}}\right) .
\end{aligned}
$$

A conformal partial wave with spinning external operators is thus labelled by two threecomponent vectors $\mathbf{n}=\left(n_{1}, n_{2}, n\right)$ and $\mathbf{m}=\left(m, m_{3}, m_{4}\right)$,

$$
\begin{aligned}
& W_{\Delta, s}^{\mathbf{n}, \mathbf{m}}\left(y_{i}\right)+\text { shadow } \\
& =\kappa_{d-\Delta, s} \frac{\gamma_{\tau, s} \bar{\gamma}_{\tau, s}}{\pi^{d / 2}} \int d^{d} y\left\langle\left\langle\mathcal{O}_{\Delta_{1}, s_{1}}\left(y_{1}\right) \mathcal{O}_{\Delta_{2}, s_{2}}\left(y_{2}\right) \mathcal{O}_{\Delta, s}(y)\right\rangle\right\rangle^{(\mathbf{n})} \\
& \quad \times\left\langle\left\langle\tilde{\mathcal{O}}_{\Delta, s}(y) \mathcal{O}_{\Delta_{3}, s_{3}}\left(y_{3}\right) \mathcal{O}_{\Delta_{4}, s_{4}}\left(y_{4}\right)\right\rangle\right\rangle^{(\mathbf{m})},
\end{aligned}
$$

where, by applying the definition (2.12) of the operation $\langle\langle\bullet\rangle\rangle$,

$$
\left\langle\left\langle\mathcal{O}_{\Delta_{1}, s_{1}}\left(y_{1}\right) \mathcal{O}_{\Delta_{2}, s_{2}}\left(y_{2}\right) \mathcal{O}_{\Delta_{3}, s_{3}}\left(y_{3}\right)\right\rangle\right\rangle^{(\mathbf{n})}=\frac{\mathrm{Y}_{1}^{s_{1}-n_{2}-n} \mathrm{Y}_{2}^{s_{2}-n-n_{1}} \mathrm{Y}_{3}^{s-n_{1}-n_{2}} \mathrm{H}_{1}^{n_{1}} \mathrm{H}_{2}^{n_{2}} \mathrm{H}_{3}^{n}}{\left(y_{12}^{2}\right)^{\frac{\tau_{1}+\tau_{2}-\tau}{2}}\left(y_{23}^{2}\right)^{\frac{\tau_{2}+\tau-\tau_{1}}{2}}\left(y_{31}^{2}\right)^{\frac{\tau+\tau_{1}-\tau_{2}}{2}}} .
$$

In the same way, the shadow contribution can be projected out by introducing a contour integral as in (2.13).

\subsubsection{Spinning conserved conformal partial waves}

Conservation of external operators places additional constraints on conformal partial waves, which is a consequence of the conservation conditions on three-point functions of conserved operators $[87,88]$. The latter relates the coefficients $c_{s_{1}, s_{2}, s_{3}}^{n_{1}, n_{2}, n_{3}}$ in a general spinning threepoint function (2.17) amongst each other, reducing the number of independent forms to [85]

$$
\begin{array}{r}
\mathcal{N}\left(s_{1}, s_{2}, s_{3}\right)=1+\min \left\{s_{1}, s_{2}, s_{3}\right\}, \\
{ }^{8} \text { To be more precise, } \sum_{n_{i}}=\sum_{n_{3}=0}^{\min \left\{s_{1}, s_{2}\right\} \min \left\{s_{1}-n_{3}, s_{3}\right\}} \sum_{n_{2}=0}^{\min \left\{s_{2}-n_{3}, s_{3}-n_{2}\right\}} \sum_{n_{1}=0} .
\end{array}
$$

Let us also note that it is from this that one obtains the counting (2.16): $\sum_{n_{i}} 1=N\left(s_{1}, s_{2}, s_{3}\right)$.

${ }^{9}$ Note that our conventions differ from those in [86], which uses $H_{j k}$ and $V_{i, j k}$ in place of our $\mathrm{H}_{i}$ and $\mathrm{Y}_{i}$ above. They are related by: $\mathrm{H}_{i}=H_{j k} / P_{j k}^{2}$ and $\mathrm{Y}_{i}=\frac{P_{j k}}{P_{i k} P_{i j}} V_{i, j k}$. 
when each operator in the three-point function is conserved. The general form for a three-point function of conserved operators in $d>3$ is given as a generating functional by $[89,90],{ }^{10}$

$$
\begin{gathered}
\left\langle\mathcal{J}_{s_{1}}\left(y_{1}\right) \mathcal{J}_{s_{2}}\left(y_{2}\right) \mathcal{J}_{s_{3}}\left(y_{3}\right)\right\rangle=\sum_{k=0}^{\frac{1+\min \left(s_{1}, s_{2}, s_{3}\right)}{2}} \mathrm{c}_{\mathcal{J}_{s_{1}} \mathcal{J}_{s_{2}} \mathcal{J}_{s_{3}}{ }_{2} F_{1}\left(\frac{1}{2}-k,-k, 3-\frac{d}{2}-2 k,-\frac{1}{2} \frac{\Lambda}{\mathrm{H}_{1}^{2} \mathrm{H}_{2}^{2} \mathrm{H}_{3}^{2}}\right)} \\
\times \frac{e^{\mathrm{Y}_{1}+\mathrm{Y}_{2}+\mathrm{Y}_{3}} F_{0}\left(\frac{d}{2}+2 k-1,-\frac{1}{2} \mathrm{H}_{1}\right)_{0} F_{1}\left(\frac{d}{2}+2 k-1,-\frac{1}{2} \mathrm{H}_{2}\right)_{0} F_{1}\left(\frac{d}{2}+2 k-1,-\frac{1}{2} \mathrm{H}_{3}\right)}{\left(y_{12}^{2}\right)^{\frac{d}{2}-1}\left(y_{23}^{2}\right)^{\frac{d}{2}-1}\left(y_{31}^{2}\right)^{\frac{d}{2}-1}} \Lambda^{2 k},
\end{gathered}
$$

with

$$
\Lambda=\mathrm{Y}_{1} \mathrm{Y}_{2} \mathrm{Y}_{3}+\frac{1}{2}\left[\mathrm{Y}_{1} \mathrm{H}_{1}+\mathrm{Y}_{2} \mathrm{H}_{2}+\mathrm{Y}_{3} \mathrm{H}_{3}\right]
$$

and $k$ takes both integer and half integer values. The OPE coefficients $c_{\mathcal{J}_{s_{1}} \mathcal{J}_{s_{2}} \mathcal{J}_{s_{3}}}^{k}$ are not fixed by current conservation and depend on the theory.

The above counting implies, for instance, that conserved conformal partial waves representing the contribution of a conserved primary operator are labelled by two half-integers $k \in\left\{0,1 / 2,1, \ldots, 1+\min \left(s_{1}, s_{2}, s\right) / 2\right\}$ and $\tilde{k} \in\left\{0,1 / 2,1, \ldots, 1+\min \left(s, s_{3}, s_{4}\right) / 2\right\}$, where $\left(s_{1}, s_{2}, s_{3}, s_{4}\right)$ are the spins of the external conserved operators,

$$
\begin{aligned}
& \mathcal{W}_{\left(s_{1}, s_{2}|s| s_{3}, s_{4}\right)}^{k, \tilde{k}}\left(y_{i}\right)+\text { shadow } \\
& \quad=\kappa_{d-\Delta, s} \frac{\gamma_{\tau, s} \bar{\gamma}_{\tau, s}}{\pi^{d / 2}} \int d^{d} y\left\langle\left\langle\mathcal{J}_{s_{1}}\left(y_{1}\right) \mathcal{J}_{s_{2}}\left(y_{2}\right) \mathcal{J}_{s}(y)\right\rangle\right\rangle^{(k)}\left\langle\left\langle\tilde{\mathcal{J}}_{s}(y) \mathcal{J}_{s_{3}}\left(y_{3}\right) \mathcal{J}_{s_{4}}\left(y_{4}\right)\right\rangle\right\rangle^{(\tilde{k})}
\end{aligned}
$$

where $\mathcal{J}_{s}$ is the exchanged spin- $s$ conserved current and

$$
\begin{aligned}
& \left\langle\left\langle\mathcal{J}_{s_{1}}\left(y_{1}\right) \mathcal{J}_{s_{2}}\left(y_{2}\right) \mathcal{J}_{s}\left(y_{3}\right)\right\rangle\right\rangle^{(k)}={ }_{2} F_{1}\left(\frac{1}{2}-k,-k, 3-\frac{d}{2}-2 k,-\frac{1}{2} \frac{\Lambda}{\mathrm{H}_{1}^{2} \mathrm{H}_{2}^{2} \mathrm{H}_{3}^{2}}\right) \\
& \times \frac{e^{\mathrm{Y}_{1}+\mathrm{Y}_{2}+\mathrm{Y}_{3}}{ }_{0} F_{1}\left(\frac{d}{2}+2 k-1,-\frac{1}{2} \mathrm{H}_{1}\right)_{0} F_{1}\left(\frac{d}{2}+2 k-1,-\frac{1}{2} \mathrm{H}_{2}\right)_{0} F_{1}\left(\frac{d}{2}+2 k-1,-\frac{1}{2} \mathrm{H}_{3}\right)}{\left(y_{12}^{2}\right)^{\frac{d}{2}-1}\left(y_{23}^{2}\right)^{\frac{d}{2}-1}\left(y_{31}^{2}\right)^{\frac{d}{2}-1}} \Lambda^{2 k}
\end{aligned}
$$

Conservation of higher-spin currents is a powerful constraint, with the presence of a single exactly conserved current of spin $s>2$ in the spectrum implying (in $d \geq 3$ ) that the theory is a free one [91-95]. ${ }^{11}$ In this case the label $k$ of each independent structure in (2.23) denotes the spin of the free conformal representation [90]. An example which we employ later on is the free scalar, where $k=0$ (the scalar singleton) and the corresponding

\footnotetext{
${ }^{10}$ To extract the explicit structure of the correlator from the generating function form (2.23) one expands and collects monomials of the form $Y_{1}^{s_{1}-n_{2}-n_{3}} Y_{2}^{s_{2}-n_{1}-n_{3}} Y_{3}^{s-n_{1}-n_{2}} H_{1}^{n_{1}} H_{2}^{n_{2}} \mathrm{H}_{3}^{n_{3}}$.

${ }^{11}$ Assuming a single stress tensor.
} 
conserved three-point structure can be conveniently expressed in terms of Bessel functions ${ }^{12}$

$$
\left\langle\mathcal{J}_{s_{1}}\left(y_{1}\right) \mathcal{J}_{s_{2}}\left(y_{2}\right) \mathcal{J}_{s_{3}}\left(y_{3}\right)\right\rangle=\mathrm{c}_{\mathcal{J}_{s_{1}} \mathcal{J}_{s_{2}} \mathcal{J}_{s_{3}}}^{0} \frac{\left(\prod_{i=1}^{3} 2^{\frac{d}{4}-1} q_{i}^{\frac{1}{2}-\frac{d-2}{4}} \Gamma\left(\frac{d-2}{2}\right) J_{\frac{d}{2}-2}\left(\sqrt{q_{i}}\right)\right) \mathrm{Y}_{1}^{s_{1}} Y_{2}^{s_{2}} Y_{3}^{s_{3}}}{\left(y_{12}^{2}\right)^{d / 2-1}\left(y_{23}^{2}\right)^{d / 2-1}\left(y_{31}^{2}\right)^{d / 2-1}}
$$

where $q_{i}=2 \mathrm{H}_{i} \partial_{Y_{i+1}} \cdot \partial_{\Upsilon_{i+2}}$. The OPE coefficients were worked out in [47] to be

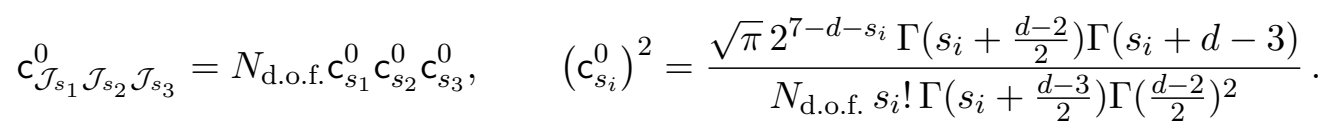

\section{CPWE of spinning Witten diagrams}

The integral representation of the CPWE is most suitable for establishing CPWEs of Witten diagrams, as it arises naturally from their harmonic function decomposition (see [37] for a detailed review):

The analogue of the CPWE expansion in the bulk is the decomposition into partial waves of the AdS isometry group. I.e. in terms of harmonic functions with energy and spin quantum numbers, ${ }^{13}$

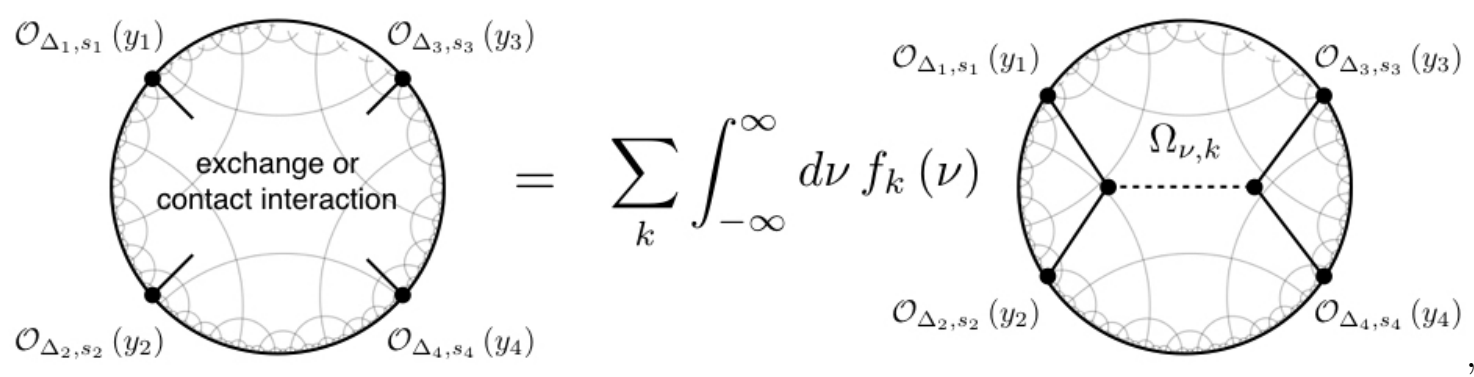

To make contact with the CPWE on the boundary, one notes that harmonic functions factorise [43]

$$
\Omega_{\nu, k}\left(x_{1}, u_{1} ; x_{2}, u_{2}\right)=\frac{\nu^{2}}{\pi k !\left(\frac{d}{2}-1\right)_{k}} \int_{\partial \mathrm{AdS}} d^{d} y \Pi_{\frac{d}{2}+i \nu, k}\left(x_{1}, u_{1} ; y, \hat{\partial}_{z}\right) \Pi_{\frac{d}{2}-i \nu, k}\left(y, z ; x_{2}, u_{2}\right),
$$

${ }^{12}$ To see this one employs the identity

$$
\Gamma(\alpha+1) x^{-\alpha} J_{\alpha}(2 x)=2_{0}^{-\alpha} F_{1}\left(\alpha+1 ;-\frac{x^{2}}{4}\right) .
$$

${ }^{13}$ The harmonic function $\Omega_{\nu, s-2 k}$ is a symmetric and traceless (in both sets of indices) spin $s-2 k$ Eigenfunction of the Laplacian,

$$
\left(\square+\left(\frac{d}{2}+i \nu\right)\left(\frac{d}{2}-i \nu\right)+s-2 k\right) \Omega_{\nu, s-2 k}=0,
$$

which is divergence-free, $\nabla \cdot \Omega_{\nu, s-2 k}=0$. 
into a product of two boundary-to-bulk propagators of dimensions $\frac{d}{2} \pm i \nu$ and the same spin $k$. We see that, like for conformal partial waves (section 2 equation (2.14)), each bulk partial wave factorises into a product of two three-point Witten diagrams,

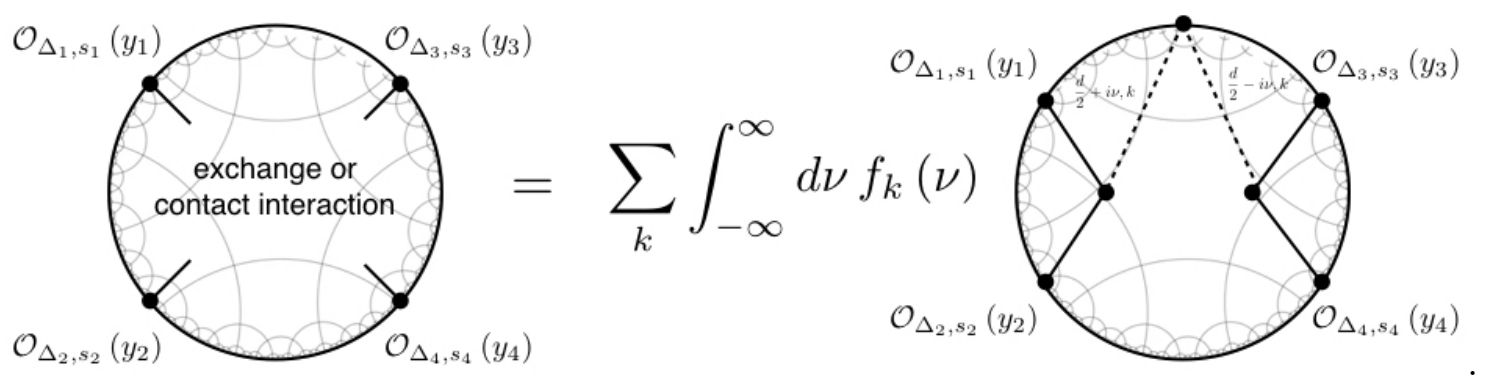

Evaluating the bulk integrals yields a decomposition of the Witten diagram into products of three-point conformal structures on the boundary - i.e. the integral representation (2.14) of the conformal partial wave expansion.

So far this approach has been applied to compute the CPWEs of tree-level Witten diagrams with only external scalars. This includes: the exchange of a massive spin- $s$ field and the graviton exchange [40]; ${ }^{14}$ the exchange of spin- $s$ gauge field on $\operatorname{AdS}_{d+1}$ [45] and contact diagrams for a general quartic scalar self-interaction [31].

\section{Spinning Exchange Witten Diagrams}

In this section we generalise the aforementioned results, to include all possible four-point exchange diagrams involving totally symmetric fields of arbitrary integer spin and mass both internally and externally. ${ }^{15}$ To wit, we decompose into conformal partial waves the following general exchange of a spin- $s$ field of mass $m^{2} R^{2}=\Delta(\Delta-d)-s$ in $\operatorname{AdS}_{d+1}$

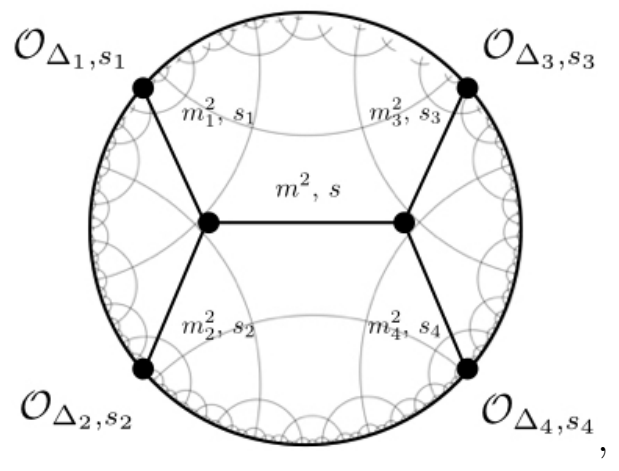

between external fields of spin $s_{i}$ and mass $m_{i}^{2} R^{2}=\Delta_{i}\left(\Delta_{i}-d\right)-s_{i}$.

\footnotetext{
${ }^{14}$ See also the very recent [66] which also employed this approach to compute the CPWE of four-point exchange Witten diagrams with external scalars, but using a different form for the cubic vertex. Since the latter vertex is equivalent to the ones used in $[40,45]$ up to total derivatives, the result is the same up to contact terms.

${ }^{15}$ For other works on spinning exchange diagrams, see: $[96,97]$ in the context of higher-spin gauge theories and more recently [64] in the context of the geodesic Witten diagram decomposition of standard Witten diagrams.
} 
The first step is to obtain the decomposition (3.2) of the exchange diagram. This is achieved by expressing the bulk-to-bulk propagator of the exchanged field in a basis of harmonic functions [40, 45, 98], which we review for massive fields in section 3.2.1 and for massless fields in section 3.2.2. This leads to the decomposition (3.4) of the exchange diagram (3.5) into products of tree-level three-point Witten diagrams, whose evaluation leads to the sought-for conformal partial wave expansion via identification with the integral form (2.20) of the conformal partial waves.

\subsection{Spinning three-point Witten diagrams}

In the light of the decomposition (3.2) of Witten diagrams, a key step to obtain CPWEs of spinning diagrams is therefore the evaluation of tree-level three-point Witten diagrams involving fields of arbitrary integer spin and mass. For parity even theories, this was carried out in [47] in general dimensions, whose results we review here and also further supplement with new ones.

\subsubsection{Building blocks of cubic vertices}

Employing the ambient space formalism (reviewed in appendix A), a convenient basis of on-shell cubic vertices between totally symmetric fields $\varphi_{s_{i}}$ of spins $s_{i}$ and mass $m_{i}^{2} R^{2}=$ $\Delta_{i}\left(\Delta_{i}-d\right)-s_{i}$ is given by

$$
\begin{aligned}
I_{s_{1}, s_{2}, s_{3}}^{n_{1}, n_{2}, n_{3}}=\mathcal{Y}_{1}^{s_{1}-n_{2}-n_{3}} \mathcal{Y}_{2}^{s_{2}-n_{3}-n_{1}} \mathcal{Y}_{3}^{s_{3}-n_{1}-n_{2}} & \\
& \times\left.\mathcal{H}_{1}^{n_{1}} \mathcal{H}_{2}^{n_{2}} \mathcal{H}_{3}^{n_{3}} \varphi_{s_{1}}\left(X_{1}, U_{1}\right) \varphi_{s_{2}}\left(X_{2}, U_{2}\right) \varphi_{s_{s}}\left(X_{3}, U_{3}\right)\right|_{X_{i}=X},
\end{aligned}
$$

which is parameterised by the six basic contractions

$$
\begin{array}{lll}
\mathcal{Y}_{1}=\partial_{U_{1}} \cdot \partial_{X_{2}}, & \mathcal{Y}_{2}=\partial_{U_{2}} \cdot \partial_{X_{3}}, & \mathcal{Y}_{3}=\partial_{U_{3}} \cdot \partial_{X_{1}}, \\
\mathcal{H}_{1}=\partial_{U_{2}} \cdot \partial_{U_{3}}, & \mathcal{H}_{2}=\partial_{U_{3}} \cdot \partial_{U_{1}}, & \mathcal{H}_{3}=\partial_{U_{1}} \cdot \partial_{U_{2}} .
\end{array}
$$

Recall that, in accordance with standard AdS/CFT lore, the basis elements (3.6) are in one-to-one correspondence with the independent three-point conformal structures (2.21).

The most general cubic vertex thus takes the form (cf. footnote 8 for the sum over $n_{i}$ )

$$
V_{s_{1}, s_{2}, s_{3}}=\sum_{n_{i}} g_{s_{1}, s_{2}, s_{3}}^{n_{1}, n_{2}, n_{3}} I_{s_{1}, s_{2}, s_{3}}^{n_{1}, n_{2}, n_{3}}
$$

The choice of basis (3.6) is convenient for three main reasons:

1. Simplicity: The basis is built from the (commuting) ambient partial derivatives as opposed to the (non-commuting) AdS covariant derivatives.

2. Ease of manipulation and computation: This is a consequence of the above simplicity. One important example is given by integration by parts in the ambient formalism. While this is in general more involved compared to standard integration by parts directly on the AdS manifold, the basis (3.6) makes integration by parts as simple as in flat space. See [99] for details on integration by parts in the ambient space framework, and in particular for the basis (3.6). 
3. Physical interpretation: Any vertex expressed in terms of covariant derivatives can straightforwardly be cast in terms of the basis (3.6), and vice versa, using (see section A)

$$
\nabla_{A}=\mathcal{P}_{A}^{B} \frac{\partial}{\partial X^{B}}-\frac{X^{B}}{X^{2}} \Sigma_{A B}
$$

where

$$
\Sigma_{A B}=U_{[A} \frac{\partial}{\left.\partial U^{B}\right]}=U_{A} \frac{\partial}{\partial U^{B}}-U_{B} \frac{\partial}{\partial U^{A}},
$$

is the spin connection in the ambient generating function formalism. See appendix B of [47] for more details about radial reduction.

\subsubsection{Spinning Witten diagrams from a scalar seed}

Another virtue of the ambient space formalism is that Witten diagrams with spinning external legs can be seamlessly generated from those with only external scalars (which are comparably straightforward to evaluate) via the application of appropriate differential operators in the boundary variables. The ease of this approach to spinning Witten diagrams within the ambient framework is owing in particular to the homogeneity of the ambient representatives in both the bulk and boundary coordinates. The implication of this observation for the three-point Witten diagram generated by the basis vertex (3.6) is that it can be re-expressed in the form
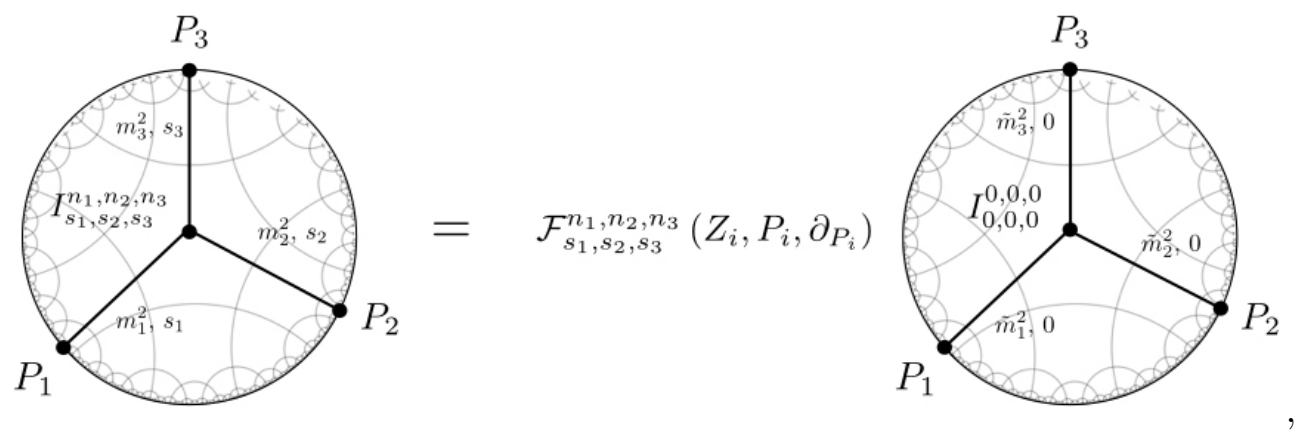

for some homogeneous differential operator $\mathcal{F}_{s_{1}, s_{2}, s_{3}}^{n_{1}, n_{2}, n_{3}}\left(Z_{i}, P_{i}, \partial_{P_{i}}\right)$, acting on the diagram generated by the coupling $I_{0,0,0}^{0,0,0}$ between scalars of some mass $\tilde{m}_{i}^{2}$. The latter is a well known integral which is straightforward to evaluate [100], which we review in section D. Naturally, since the action of $\mathcal{F}_{s_{1}, s_{2}, s_{3}}^{n_{1}, n_{2}}$ increases the spin of the external legs, it will be a non-trivial function of $Z_{i}$.

The decomposition (3.11) of the spinning three-point Witten diagram can straightforwardly be obtained by noting that spinning bulk-to-boundary propagators have an analo- 
gous differential relationship to scalar bulk-to-boundary propagators $[37]^{16}$

$$
K_{\Delta, s}(X, U ; P, Z)=\frac{1}{(\Delta-1)_{s}}\left(\mathcal{D}_{P}(Z ; U)\right)^{s} K_{\Delta, 0}(X ; P),
$$

with differential operator

$$
\mathcal{D}_{P}(Z ; U)=(Z \cdot U)\left(Z \cdot \frac{\partial}{\partial Z}-P \cdot \frac{\partial}{\partial P}\right)+(P \cdot U)\left(Z \cdot \frac{\partial}{\partial P}\right) .
$$

Ambient partial derivatives of spinning bulk-to-boundary propagators, which arise naturally from the basis (3.6), can readily be expressed in a similar form:

$$
\left(U_{j} \cdot \partial_{X}\right)^{n} K_{\Delta, s}\left(X, U_{i} ; P, Z\right)=\frac{1}{(\Delta-1)_{s}}\left(\mathcal{D}_{P}(Z ; U)\right)^{s}\left(U_{i} \cdot \partial_{X}\right)^{n} K_{\Delta, 0}(X ; P)
$$

with

$$
\left(U_{i} \cdot \partial_{X}\right)^{n} K_{\Delta, 0}(X ; P)=2^{n}\left(\Delta+1-\frac{d}{2}\right)_{n}\left(U_{i} \cdot P\right)^{n} K_{\Delta+n, 0}(X ; P) .
$$

This further illustrates the convenience of the choice of basis (3.6).

Employing the expression for spinning bulk-to-boundary propagators (3.16) one then obtains

$$
\begin{gathered}
\mathcal{F}_{s_{1}, s_{2}, s_{3}}^{n_{1}, n_{2}, n_{3}}=\frac{2^{\tilde{s}_{1}+\tilde{s}_{2}+\tilde{s}_{3}}\left(\Delta_{1}+1-\frac{d}{2}\right)_{\tilde{s}_{3}}\left(\Delta_{2}+1-\frac{d}{2}\right)_{\tilde{s}_{1}}\left(\Delta_{3}+1-\frac{d}{2}\right)_{\tilde{s}_{2}}}{\left(\Delta_{1}-1\right)_{s_{1}}\left(\Delta_{2}-1\right)_{s_{2}}\left(\Delta_{3}-1\right)_{s_{3}}\left(\tilde{s}_{1}\right) !\left(\tilde{s}_{2}\right) !\left(\tilde{s}_{3}\right) !} \\
\times \mathcal{H}_{1}^{n_{1}} \mathcal{H}_{2}^{n_{2}} \mathcal{H}_{3}^{n_{3}} \overline{\mathcal{H}}_{1}^{\tilde{s}_{2}} \overline{\mathcal{H}}_{2}^{\tilde{s}_{3}} \overline{\mathcal{H}}_{3}^{\tilde{s}_{1}} \mathcal{D}_{P_{1}}^{s_{1}} \mathcal{D}_{P_{2}}^{s_{2}} \mathcal{D}_{P_{3}}^{s_{3}}\left(\bar{U}_{1} \cdot P_{1}\right)^{\tilde{s}_{3}}\left(\bar{U}_{2} \cdot P_{2}\right)^{\tilde{s}_{2}}\left(\bar{U}_{3} \cdot P_{3}\right)^{\tilde{s}_{1}}
\end{gathered}
$$

where for concision we defined $\tilde{s}_{i}=s_{i}-n_{i-1}-n_{i+1}$ and introduced the auxiliary vector $\bar{U}_{i}$ which enters the contraction $\overline{\mathcal{H}}_{i}=\partial_{U_{i-1}} \cdot \partial_{\bar{U}_{i+1}}$. The mass of each scalar entering the seed vertex on the r.h.s. of (3.11) is given in terms of the quantum numbers of the original spinning fields on the l.h.s.

$$
\tilde{m}_{i}^{2} R^{2}=\tilde{\Delta}_{i}\left(\tilde{\Delta}_{i}-d\right) \quad \text { with } \quad \tilde{\Delta}_{i}=\Delta_{i}+s_{i+2}-n_{i}-n_{i+1} .
$$

What remains to obtain the result for the spinning Witten diagram in the l.h.s. of (3.11) is to simply insert the result (D.5) for the scalar seed on the r.h.s. and then act with the differential operator (3.18). Denoting the amplitude by $A_{s_{1}, s_{2}, s_{3} ; \tau_{1}, \tau_{2}, \tau_{3}}^{n_{1}, n_{2}, n_{3}}$, this procedure yields ${ }^{17}$

${ }^{16} \mathrm{By}$ evaluating the action of the differential operator one recovers the standard expression [101]

$$
K_{\Delta, s}(X, U ; P, Z)=(U \cdot \mathcal{P} \cdot Z)^{s} \frac{C_{\Delta, s}}{(-2 X \cdot P)^{\Delta}}, \quad C_{\Delta, s}=\frac{(s+\Delta-1) \Gamma(\Delta)}{2 \pi^{d / 2}(\Delta-1) \Gamma\left(\Delta+1-\frac{d}{2}\right)} .
$$

This also dictates the normalisation of the dual operator two-point function at large $N_{\text {d.o.f }}$

$$
\left\langle\mathcal{O}_{\Delta, s}\left(y_{1} ; z_{1}\right) \mathcal{O}_{\Delta, s}\left(y_{2} ; z_{2}\right)\right\rangle=\frac{C_{\Delta, s}}{\left(y_{12}^{2}\right)^{\Delta}}\left(z_{1} \cdot z_{2}+\frac{2 z_{1} \cdot y_{12} z_{2} \cdot y_{21}}{y_{12}^{2}}\right)^{s} \text {. }
$$

${ }^{17}$ The summation symbol is defined as:

$$
\sum_{\alpha, \beta, \delta, \omega, \gamma} \equiv \sum_{\alpha_{\kappa}=0}^{s_{\kappa}-k_{\kappa}} \sum_{\beta_{\kappa}=0}^{k_{\kappa}} \sum_{\delta_{\kappa}=0}^{n_{\kappa}} \sum_{\omega_{\kappa}=0}^{\alpha_{\kappa}-1+\beta_{\kappa-1}} \sum_{\gamma_{\kappa}=0}^{\alpha_{\kappa-1}+\beta_{\kappa-1}} .
$$


$A_{s_{1}, s_{2}, s_{3} ; \tau_{1}, \tau_{2}, \tau_{3}}^{n_{1}, n_{2}, n_{3}}\left(y_{1}, y_{2}, y_{3}\right)=$

$\mathrm{P}_{3} \sum_{\alpha, \beta, \delta, \omega, \gamma} \prod_{i=1}^{3}(-1)^{s_{i}-n_{i}-\delta_{i}+\alpha_{i}+\beta_{i}} 2^{s_{i}-n_{i}-\gamma_{i}-\delta_{i}-\omega_{i}} \frac{n_{i} !\left(\alpha_{i}+\beta_{i}\right) !\left(s_{i}-n_{i+1}-n_{i-1}\right) !}{\gamma_{i} ! \delta_{i} ! \alpha_{i} ! \omega_{i} !\left(\beta_{i}+\delta_{i+1}-n_{i+1}+1\right) !}$

$\times \frac{\left(\alpha_{i}+\beta_{i}+\Delta_{i}\right)_{s_{i}+\delta_{i(i+1)}-\gamma_{i+1}-n_{i+1}-\omega_{i+1}-\Delta_{i}}}{\left(\alpha_{i}+\beta_{i}-\gamma_{i+1}-\gamma_{i-1}-\omega_{i+1}+1\right) !\left(s_{i}-\alpha_{i}-n_{i+1}-n_{i-1}-\omega_{i-1}+1\right) !\left(n_{i+1}+n_{i-1}-\beta_{i}-\delta_{i+1}-\delta_{i-1}+1\right) !}$

$\times \mathrm{H}_{1}^{\gamma_{1}+\delta_{1}+\omega_{1}} \mathrm{H}_{2}^{\gamma_{2}+\delta_{2}+\omega_{2}} \mathrm{H}_{3}^{\gamma_{3}+\delta_{3}+\omega_{3}} Y_{1}^{s_{1}-\gamma_{2}-\gamma_{3}-\delta_{2}-\delta_{3}-\omega_{2}-\omega_{3}} Y_{2}^{s_{2}-\gamma_{1}-\gamma_{3}-\delta_{1}-\delta_{3}-\omega_{1}-\omega_{3}} Y_{3}^{s_{3}-\gamma_{1}-\gamma_{2}-\delta_{1}-\delta_{2}-\omega_{1}-\omega_{2}}$,

where $i \cong i+3$.

The pre-factor is given by

$\mathrm{P}_{3}=\frac{1}{16 \pi^{d}} \frac{1}{\left(y_{12}\right)^{\delta_{12}}\left(y_{23}\right)^{\delta_{23}}\left(y_{31}\right)^{\delta_{31}}} \Gamma\left(\sum_{\alpha}\left(\frac{\tau_{\alpha}}{2}+s_{\alpha}-n_{\alpha}\right)-\frac{d}{2}\right) \prod_{i=1}^{3} \frac{\Gamma\left(\Delta_{i}-1\right)\left(\Delta_{i}+s_{i}-1\right)}{\Gamma\left(\Delta_{i}+1-\frac{d}{2}\right)}$,

where

$$
\delta_{(i-1)(i+1)}=\frac{1}{2}\left(\tau_{i-1}+\tau_{i+1}-\tau_{i}\right), \quad \tau_{i}=\Delta_{i}-s_{i} .
$$

While the basis (3.6) is convenient as a means to evaluate spinning Witten diagrams, the resulting one-to-one map (3.21) between the bulk basis elements (3.6) and the canonical basis (2.21) of three-point conformal structures is rather involved. In the following section we introduce an alternative bulk and boundary pair of bases, through which the aforementioned bulk-to-boundary mapping simplifies dramatically and moreover allows to elegantly re-sum the expression (3.21).

\subsubsection{A natural basis of cubic structures in AdS/CFT}

Let us motivate this alternative basis with a simple example. As observed in [47], the amplitude generated by the highest derivative basis vertex

$$
I_{s_{1}, s_{2}, s_{3}}^{0,0,0}=\left.\mathcal{Y}_{1}^{s_{1}} \mathcal{Y}_{2}^{s_{2}} \mathcal{Y}_{3}^{s_{3}} \varphi_{s_{1}}\left(X_{1}, U_{1}\right) \varphi_{s_{2}}\left(X_{2}, U_{2}\right) \varphi_{s_{s}}\left(X_{3}, U_{3}\right)\right|_{X_{i}=X}
$$

admits a very simple re-summation in terms of Bessel functions

$$
\begin{aligned}
& A_{s_{1}, s_{2}, s_{3} ; \tau_{1}, \tau_{2}, \tau_{3}}^{0,0,0}\left(y_{1}, y_{2}, y_{3}\right)=\frac{B_{s_{i} ; \tau_{i}}}{\left(y_{12}\right)^{\delta_{12}}\left(y_{23}\right)^{\delta_{23}\left(y_{31}\right)^{\delta_{31}}}} \\
& \quad \times\left[\prod_{i=1}^{3} 2^{\frac{\delta_{(i+1)(i-1)}}{2}-1} \Gamma\left(\frac{\delta_{(i+1)(i-1)}}{2}\right) q_{i}^{\frac{1}{2}-\frac{\delta_{(i+1)(i-1)}}{4}} J_{\left(\delta_{(i+1)(i-1)}-2\right) / 2}\left(\sqrt{q_{i}}\right)\right] \mathrm{Y}_{1}^{s_{1}} Y_{2}^{s_{2}} Y_{3}^{s_{3}}
\end{aligned}
$$

where we recall that $q_{i}=2 \mathrm{H}_{i} \partial_{\mathbf{Y}_{i+1}} \partial_{\boldsymbol{Y}_{i-1}}$ and the overall coefficient is given by

$$
\begin{aligned}
B_{s_{i} ; \tau_{i}}= & \frac{1}{16 \pi^{d}} \Gamma\left(\frac{\tau_{1}+\tau_{2}+\tau_{3}-d+2\left(s_{1}+s_{2}+s_{3}\right)}{2}\right) \\
& \times \prod_{i=1}^{3} \frac{(-2)^{s_{i}} \Gamma\left(s_{i}+\delta_{i(i+1)}\right) \Gamma\left(s_{i}+\delta_{(i-1) i}\right) \Gamma\left(s_{i}+\tau_{i}-1\right)}{\Gamma\left(s_{i}+\tau_{i}-\frac{d}{2}+1\right) \Gamma\left(\delta_{(i+1)(i-1)}\right) \Gamma\left(2 s_{i}+\tau_{i}-1\right)} .
\end{aligned}
$$


Such three-point conformal structures are for instance generated in free scalar CFTs (see e.g. (2.28) for the case of three-point functions of conserved operators).

Given the simplicity and compactness of the three-point conformal structure (3.24) generated by the basis vertex (3.23), it is temping to consider the following basis of conformal structures,

$$
\begin{aligned}
& \left.\left[\left[\mathcal{O}_{\Delta_{1}, s_{1}}\left(y_{1}\right) \mathcal{O}_{\Delta_{2}, s_{2}}\left(y_{2}\right) \mathcal{O}_{\Delta_{3}, s_{3}}\left(y_{3}\right)\right]\right]\right]^{(\mathbf{n})} \equiv \\
& \quad \frac{\mathrm{H}_{1}^{n_{1}} \mathrm{H}_{2}^{n_{2}} \mathrm{H}_{3}^{n_{3}}}{\left(y_{12}\right)^{\delta_{12}}\left(y_{23}\right)^{\delta_{23}}\left(y_{31}\right)^{\delta_{31}}}\left[\prod_{i=1}^{3} 2^{\frac{\delta_{(i+1)(i-1)}}{2}+n_{i}-1} \Gamma\left(\frac{\delta_{(i+1)(i-1)}}{2}+n_{i}\right)\right] \\
& \quad \times\left[\prod_{i=1}^{3} q_{i}^{\frac{1-n_{i}}{2}-\frac{\delta_{(i+1)(i-1)}}{4}} J_{\left(\delta_{(i+1)(i-1)}+2 n_{i}-2\right) / 2}\left(\sqrt{q_{i}}\right)\right] \mathrm{Y}_{1}^{s_{1}-n_{2}-n_{3}} \mathrm{Y}_{2}^{s_{2}-n_{3}-n_{1}} \mathrm{Y}_{3}^{s_{3}-n_{1}-n_{2}}
\end{aligned}
$$

in the view of simplifying the map between bulk and boundary structures.

Indeed, working iteratively one finds that the conformal structure (3.26) is generated by the bulk vertex ${ }^{18}$

$$
\mathcal{I}_{s_{1}, s_{2}, s_{3}}^{n_{1}, n_{2}, n_{3}}=\sum_{m_{i}} C_{s_{1}, s_{2}, s_{3} ; m_{1}, m_{2}, m_{3}}^{n_{1}, n_{2}, n_{3}} I_{s_{1}, s_{2}, s_{3}}^{m_{1}, m_{2}, m_{3}}
$$

with coefficients $C_{s_{1}, s_{2}, s_{3} ; m_{1}, m_{2}, m_{3}}^{n_{1}, n_{2}, n_{3}}$ given by

$$
\begin{aligned}
C_{s_{1}, s_{2}, s_{3} ; m_{1}, m_{2}, m_{3}}^{n_{1}, n_{2}, n_{3}}= & \left(\frac{d-2\left(s_{1}+s_{2}+s_{3}-1\right)-\left(\tau_{1}+\tau_{2}+\tau_{3}\right)}{2}\right)_{m_{1}+m_{2}+m_{3}} \\
& \times \prod_{i=1}^{3}\left[2^{m_{i}}\left(\begin{array}{c}
n_{i} \\
m_{i}
\end{array}\right)\left(n_{i}+\delta_{(i+1)(i-1)}-1\right)_{m_{i}}\right]
\end{aligned}
$$

In particular, denoting the three-point amplitude generated by each basis element (3.27) by $\mathcal{A}_{s_{1}, s_{2}, s_{3} ; \tau_{1}, \tau_{2}, \tau_{3}}^{n_{1}, n_{2}, n_{3}}$ we have: ${ }^{19}$

$$
\mathcal{A}_{s_{1}, s_{2}, s_{3} ; \tau_{1}, \tau_{2}, \tau_{3}}^{n_{1}, n_{2}}\left(y_{1}, y_{2}, y_{3}\right)=\mathrm{B}\left(s_{i} ; n_{i} ; \tau_{i}\right)\left[\left[\mathcal{O}_{\Delta_{1}, s_{1}}\left(y_{1}\right) \mathcal{O}_{\Delta_{2}, s_{2}}\left(y_{2}\right) \mathcal{O}_{\Delta_{3}, s_{3}}\left(y_{3}\right)\right]\right]^{(\mathbf{n})},
$$

with the coefficient $\mathrm{B}\left(s_{i} ; n_{i} ; \tau_{i}\right)$ given by

$$
\begin{aligned}
& \mathrm{B}\left(s_{i} ; n_{i} ; \tau_{i}\right)=\pi^{-d}(-2)^{\left(s_{1}+s_{2}+s_{3}\right)-\left(n_{1}+n_{2}+n_{3}\right)-4} \Gamma\left(\frac{\tau_{1}+\tau_{2}+\tau_{3}-d+2\left(s_{1}+s_{2}+s_{3}\right)}{2}\right) \\
& \times \prod_{i=1}^{3} \frac{\Gamma\left(s_{i}-n_{i+1}+n_{i-1}+\frac{\tau_{i}+\tau_{i+1}-\tau_{i-1}}{2}\right) \Gamma\left(s_{i}+n_{i+1}-n_{i-1}+\frac{\tau_{i}+\tau_{i-1}-\tau_{i+1}}{2}\right) \Gamma\left(s_{i}+n_{i+1}+n_{i-1}+\tau_{i}-1\right)}{\Gamma\left(s_{i}+\tau_{i}-\frac{d}{2}+1\right) \Gamma\left(2 n_{i}+\frac{\tau_{i+1}+\tau_{i-1}-\tau_{i}}{2}\right) \Gamma\left(2 s_{i}+\tau_{i}-1\right)} .
\end{aligned}
$$

Given a $\mathrm{CFT}_{d}$, the result (3.29) provides the complete holographic reconstruction of all cubic couplings involving totally symmetric fields in the putative dual theory on $\operatorname{AdS}_{d+1}$.

\footnotetext{
${ }^{18}$ For concision we define $\left.\sum_{m_{i}}=\sum_{m_{3}=0}^{\min \left\{s_{1}, s_{2}, n_{3}\right.}\right\} \sum_{m_{2}=0}^{\min \left\{s_{1}-n_{3}, s_{3}, n_{2}\right\}} \sum_{m_{1}=0}^{\min \left\{s_{2}-n_{3}, s_{3}-n_{2}, n_{1}\right\}}$.

${ }^{19}$ Note, the vertices constructed here should not be confused with those written down in $[65,66]$ in the context of geodesic Witten diagrams.
} 
Relation between bulk basis. To conclude it is useful to spell out the explicit dictionary between the building blocks (3.6), which allow to straightforwardly evaluate spinning Witten diagrams, and the basis (3.27) introduced in the previous section, which give a simple form for spinning three-point amplitudes.

Given a coupling of the form

$$
\mathcal{V}_{s_{1}, s_{2}, s_{3}}=\sum_{n_{i}} g_{s_{1}, s_{2}, s_{3}}^{n_{1}, n_{2}, n_{3}} I_{s_{1}, s_{2}, s_{3}}^{n_{1}, n_{2}, n_{3}}
$$

the problem is to determine the explicit form of the coefficient $\tilde{g}_{s_{1}, s_{2}, s_{3}}^{n_{1}, n_{2}, n_{3}}$ in the basis:

$$
\mathcal{V}_{s_{1}, s_{2}, s_{3}}=\sum_{n_{i}} \tilde{g}_{s_{1}, s_{2}, s_{3}}^{n_{1}, n_{2}, n_{3}} \mathcal{I}_{s_{1}, s_{2}, s_{3}}^{n_{1}, n_{2}, n_{3}}
$$

with $I_{s_{1}, s_{2}, s_{3}}^{n_{1}, n_{2}, n_{3}}$ and $\mathcal{I}_{s_{1}, s_{2}, s_{3}}^{n_{1}, n_{2}, n_{3}}$ given in (3.6) and (3.27), respectively. Working iteratively, one arrives at the following expression for the coefficient $\tilde{g}_{s_{1}, s_{2}, s_{3}}^{n_{1}, n_{2}}$ as a function of the coefficients $g_{s_{1}, s_{2}, s_{3}}^{n_{1}, n_{2}, n_{3}}$ in the original basis: ${ }^{20}$

$\tilde{g}_{s_{1}, s_{2}, s_{3}}^{n_{1}, n_{2}, n_{3}}=\sum_{m_{i}}\left[\frac{g_{m_{1}, m_{2}, m_{3}}}{\left(\frac{d}{2}+1+\sum_{\alpha}\left(m_{\alpha}-s_{\alpha}-\frac{\tau_{\alpha}}{2}\right)\right)_{m_{1}+m_{2}+m_{3}}} \prod_{i=1}^{3}(-1)^{n_{i}+m_{i}} \frac{\left(2 n_{i}+\delta_{j k}-1\right)}{2^{m_{i}}\left(n_{i}+\delta_{j k}-1\right)_{m_{i}+1}}\left(\begin{array}{c}m_{i} \\ n_{i}\end{array}\right)\right]$,

which is the inverse of the map (3.27).

Notice that the new basis (3.26) generalises to non-conserved operators the basis (2.26) of three-point conserved conformal structures. In this regard, our basis (3.26) seems to be naturally selected by free singleton CFTs.

\subsection{Spinning bulk-to-bulk propagators}

In this section we review previous works on the harmonic function decomposition of bulkto-bulk propagators for totally symmetric fields of arbitrary mass and integer spin [45]. ${ }^{21}$

Up to cubic order in perturbations about the AdS background, a spin- $s$ field of mass $m^{2} R^{2}=\Delta(\Delta-d)-s$ is governed by an effective Euclidean action of the form

$$
S_{m^{2}, s}\left[\varphi_{s}\right]=s ! \int_{\operatorname{AdS}} \frac{1}{2} \varphi_{s}\left(x, \partial_{u}\right)\left(\square-m^{2}+\ldots\right) \varphi_{s}(x, u)+\varphi_{s}\left(x, \partial_{u}\right) J_{s}(x, u)+\mathcal{O}\left(\varphi^{4}\right),
$$

where the source $J_{s}$ in the cubic interaction term is quadratic in the perturbations. The ... denote terms which depend on the off-shell completion, which we discuss case-by-case in the sequel.

\footnotetext{
${ }^{20}$ For concision we define:

$$
\sum_{m_{i}}=\sum_{m_{3}=n_{3}}^{\operatorname{Min}\left\{s_{1}, s_{2}\right\}} \sum_{m_{2}=n_{2}}^{\operatorname{Min}\left\{s_{3}, s_{1}-m_{3}\right\}} \sum_{m_{1}=n_{1}}^{\operatorname{Min}\left\{s_{2}-m_{3}, s_{3}-m_{2}\right\}} .
$$

${ }^{21}$ For earlier works spinning bulk-to-bulk propagators, see [102, 103] by B. Allen for the graviton and (massive and massless) vector propagators (also [40, 104-107]); for higher spin see [40, 96, 97, 108-113].
} 
Upon varying the action, the corresponding bulk-to-bulk propagator satisfies an equation of the form

$$
\left(\square_{1}-m^{2}+\ldots\right) \Pi_{m^{2}, s}\left(x_{1} ; x_{2}\right)=-\delta^{d+1}\left(x_{1}, x_{2}\right),
$$

where for convenience we suppressed the index structure, for now. To determine the propagator as a decomposition in harmonic functions, one can consider an ansatz of the form

$$
\begin{aligned}
& \Pi_{m^{2}, s}\left(x_{1}, u_{1} ; x_{2}, u_{2}\right) \\
& \quad=\sum_{k=0}^{\lfloor s / 2\rfloor} \sum_{l=0}^{s-2 k} \int_{-\infty}^{\infty} d \nu g_{k, l}(\nu)\left(u_{1}^{2}\right)^{k}\left(u_{2}^{2}\right)^{k}\left(u_{1} \cdot \nabla_{1}\right)^{l}\left(u_{2} \cdot \nabla_{2}\right)^{l} \Omega_{\nu, s-2 k-l}\left(x_{1}, u_{1} ; x_{2}, u_{2}\right) .
\end{aligned}
$$

The functions $g_{k, l}(\nu)$ are fixed by requiring that the equation of motion (3.36) is satisfied.

We first review the solution for massive spinning fields before moving on to the massless case, where one has the additional requirement of gauge invariance.

\subsubsection{Massive case}

The Lagrangian formulation for freely propagating totally symmetric massive fields of arbitrary spin was first considered by Singh and Hagen in the 70's $[114,115] .{ }^{22}$ In order for the Fierz-Pauli physical state conditions [120-122]

$$
\left(\square-m^{2}\right) \varphi_{s}(x, u)=0, \quad\left(\partial_{u} \cdot \nabla\right) \varphi_{s}(x, u)=0, \quad\left(\partial_{u} \cdot \partial_{u}\right) \varphi_{s}(x, u)=0,
$$

to be recovered upon varying the action, the field content consists of the traceless field $\varphi_{s}$, and additional traceless auxiliary fields of ranks $s-2, s-3, \ldots, 0$ which vanish on-shell. ${ }^{23}$

The complete off-shell form of the free Lagrangian is involved, and is moreover currently unavailable in its entirety on an AdS background. On the other hand, the terms which have not yet been identified explicitly are those which vanish on-shell (i.e. the ... in (3.35)) and thus only generate contact terms in exchange amplitudes. The latter are not universal contributions, as they are highly dependent on the field frame. For our purposes it is therefore not necessary to keep track of such terms, ${ }^{24}$ and we can solve the following equation for the massive spin- $s$ bulk-to-bulk propagator

$$
\left(\square_{1}-m^{2}\right) \Pi_{m^{2}, s}\left(x_{1}, u_{1} ; x_{2}, u_{2}\right)=-\left\{\left(u_{1} \cdot u_{2}\right)^{s}\right\} \delta^{d+1}\left(x_{1}, x_{2}\right),
$$

where the notation $\{\bullet\}$ signifies a traceless projection.

Since in this case the field is traceless, the following ansatz can be considered for the bulk-to-bulk propagator

$$
\Pi_{m^{2}, s}\left(x_{1}, w_{1} ; x_{2}, w_{2}\right)=\sum_{l=0}^{s} \int_{-\infty}^{\infty} d \nu g_{l}(\nu)\left(w_{1} \cdot \nabla_{1}\right)^{l}\left(w_{2} \cdot \nabla_{2}\right)^{l} \Omega_{\nu, s-l}\left(x_{1}, w_{1} ; x_{2}, w_{2}\right),
$$

\footnotetext{
${ }^{22}$ See also [116-119].

${ }^{23}$ See $[123,124]$ for an alternative formulation of the free massive Lagrangian in terms of curvatures, free from such auxiliary fields. Their removal, however, comes at the price of introducing non-localities.

${ }^{24}$ When it is feasible we do keep track of contact terms, such as for the massless case introduced in the following section.
} 
where the null auxiliary vectors $w_{i}^{2}=0$ enforce tracelessness. Substituting into the equation of motion (3.39), one finds [45]

$$
\begin{aligned}
g_{l}(\nu)= & \frac{1}{\left(\frac{d}{2}-\Delta\right)^{2}+\nu^{2}-l+l(d+2 s-\ell-1)} \\
& \times \frac{2^{l}(s-l+1)_{l}\left(\frac{d}{2}+s-l-\frac{1}{2}\right)_{l}}{l !(d+2 s-2 l-1)_{l}\left(\frac{d}{2}+s-l+i \nu\right)_{l}\left(\frac{d}{2}+s-l-i \nu\right)_{l}} .
\end{aligned}
$$

Before moving on to consider the massless case, let us briefly highlight some generic features of the propagator (3.40):

- The traceless and transverse part of the propagator (corresponding to $l=0$ in (3.40))

$$
\Pi_{m^{2}, s}^{T T}\left(x_{1} ; x_{2}\right)=\int_{-\infty}^{\infty} \frac{d \nu}{\left(\frac{d}{2}-\Delta\right)^{2}+\nu^{2}} \Omega_{\nu, s}\left(x_{1} ; x_{2}\right),
$$

is universal, and encodes the exchanged single-particle state of spin- $s$ and mass $m^{2} R^{2}=\Delta(\Delta-d)-s$.

- The remaining contributions from harmonic functions of spin $<s$ (the $l>0$ in (3.40)) are purely off-shell, and generate only contact terms in exchange amplitudes.

\subsubsection{Massless case}

On contrast to the massive case discussed in the previous section, the construction of free Lagrangians for massless fields is somewhat simplified owing to the additional guidance provided by gauge invariance.

Recalling that the concept of masslessness in AdS is slightly deformed owing to the background curvature, requiring gauge invariance of the Fierz-Pauli system (3.38) under the gauge transformation

$$
\delta_{\xi} \varphi_{s}(x, u)=(u \cdot \nabla) \xi_{s-1}(x, u)
$$

fixes $\Delta=s+d-2$ in the mass $m^{2} R^{2}=\Delta(\Delta-d)-s$. The complete off-shell Lagrangian form was determined by Fronsdal in the 70's [125], and reads

$$
S_{\text {Fronsdal }}^{(2)}\left[\varphi_{s}\right]=\frac{s !}{2} \int_{\operatorname{AdS}_{d+1}} \varphi_{s}\left(x ; \partial_{u}\right) \mathcal{G}_{s}(x ; u),
$$

where $\mathcal{G}_{s}$ is the corresponding spin- $s$ generalisation of the linearised Einstein tensor

$$
\mathcal{G}_{s}(x ; u)=\left(1-\frac{1}{4} u^{2} \partial_{u} \cdot \partial_{u}\right) \mathcal{F}_{s}\left(x ; u, \nabla, \partial_{u}\right) \varphi_{s}(x, u),
$$

with $\mathcal{F}_{s}$ the so-called Fronsdal operator

$$
\mathcal{F}_{s}\left(x, u, \nabla, \partial_{u}\right)=\square-m^{2}-u^{2}\left(\partial_{u} \cdot \partial_{u}\right)-(u \cdot \nabla)\left(\left(\nabla \cdot \partial_{u}\right)-\frac{1}{2}(u \cdot \nabla)\left(\partial_{u} \cdot \partial_{u}\right)\right) .
$$


The latter is fixed by invariance under linearised spin- $s$ gauge transformations (3.43) with symmetric and traceless rank $s-1$ gauge parameter $\xi_{s-1} \cdot{ }^{25}$ The Bianchi identity

$$
\left(\partial_{u} \cdot \nabla\right) \mathcal{G}_{s}(x, u)=0
$$

requires that the field $\varphi_{s}$ is double-traceless ${ }^{26}$

$$
\left(\partial_{u} \cdot \partial_{u}\right)^{2} \varphi_{s}(x, u)=0
$$

To determine the bulk-to-bulk propagator one needs to invert the equation of motion with source

$$
\left(1-\frac{1}{4} u^{2} \partial_{u} \cdot \partial_{u}\right) \mathcal{F}_{s}\left(x ; u, \nabla, \partial_{u}\right) \varphi_{s}(x, u)=-J_{s}(x, u),
$$

where from gauge-invariance it follows that $J_{s}$ is conserved on-shell, $\left(\partial_{u} \cdot \nabla\right) J_{s} \approx 0 .{ }^{27}$ For tree-level diagrams involving a single exchange, this inversion is independent of the off-shell gauge fixing of the exchanged field, since the exchanged field couples to on-shell external legs. In this context, the bulk-to-bulk propagator can be determined disregarding terms proportional to gradients $[96,97,112]$ — both in the equation of motion and in the solution. To wit, one may solve ${ }^{28}$

$$
\begin{aligned}
& {\left[\left(\square_{1}-m^{2}\right)-u_{1}^{2}\left(\partial_{u_{1}} \cdot \partial_{u_{1}}\right)\right] \Pi_{s}\left(x_{1}, u_{1} ; x_{2}, u_{2}\right)} \\
& \quad=-\left(1-\frac{1}{4} u_{1}^{2} \partial_{u_{1}} \cdot \partial_{u_{1}}\right)^{-1}\left\{\left\{\left(u_{1} \cdot u_{2}\right)^{s} \delta^{d+1}\left(x_{12}\right)\right\}\right\},
\end{aligned}
$$

up to gradient terms. It is then sufficient to make an ansatz that is free from gradient terms,

$$
\Pi_{s}\left(x_{1}, u_{1} ; x_{2}, u_{2}\right)=\sum_{k=0}^{\lfloor s / 2\rfloor} \int_{-\infty}^{\infty} d \nu g_{k}(\nu)\left(u_{1}^{2}\right)^{k}\left(u_{2}^{2}\right)^{k} \Omega_{\nu, s-2 k}\left(x_{1}, u_{1} ; x_{2}, u_{2}\right) .
$$

\footnotetext{
${ }^{25}$ Alternative formulations have been developed which eliminate this algebraic trace constraint on the gauge parameter, however they come at the price of introducing non-localities [126] or auxiliary fields [96, 127, 128].

${ }^{26}$ Note that the double-trace of $\varphi_{s}$ is gauge invariant owing to the tracelessness of the gauge parameter. Forgoing the double-traceless constraint (3.48) without introducing auxiliary fields (apart from deforming the Bianchi identity (3.47) and thus requiring a modification of the action (3.44)) would lead to the propagation of non-unitary modes, which one may try to kill by imposing appropriate boundary conditions. This has been shown to be possible in flat space [128] though it is not yet clear if this approach can be extended to AdS space-times, or if it is compatible with introducing a source. For this reason we stick to the standard Fronsdal formulation (3.44) with double-trace constraint (3.48).

27 To be more precise, consistency with higher-spin symmetry (3.43) requires that $J_{s}$ has vanishing double-trace, and moreover is conserved up to pure trace terms,
}

$$
\left(\partial_{u} \cdot \nabla\right) J_{s}(x, u) \approx \mathcal{O}\left(u^{2}\right) .
$$

As we shall demonstrate explicitly in section 3.4.1 (supplemented by section B), improvement terms (which do not contribute to on-shell vertices) can be added to the $J_{s}$ such that it is exactly conserved.

${ }^{28}$ The symbol $\{\{\bullet\}\}$ indicates a double-traceless projection:

$$
\left(\partial_{u} \cdot \partial_{u}\right)^{2}\{\{f(u, x)\}\}=0, \quad \text { and } \quad\{\{f(u, x)\}\}=f(u, x) \quad \text { iff } \quad\left(\partial_{u} \cdot \partial_{u}\right)^{2} f(u, x)=0 .
$$


Plugging the ansatz into (3.52) fixes the functions $g_{s, k}(\nu)[45]$

$$
\begin{aligned}
g_{s, 0}(\nu)= & \frac{1}{\left(\frac{d}{2}+s-2\right)^{2}+\nu^{2}}, \\
g_{s, k}(\nu)= & -\frac{(1 / 2)_{k-1}}{2^{2 k+3} \cdot k !} \frac{(s-2 k+1)_{2 k}}{\left(\frac{d}{2}+s-2 k\right)_{k}\left(\frac{d}{2}+s-k-3 / 2\right)_{k}} \\
& \times \frac{\left(\left(\frac{d}{2}+s-2 k+i \nu\right) / 2\right)_{k-1}\left(\left(\frac{d}{2}+s-2 k-i \nu\right) / 2\right)_{k-1}}{\left(\left(\frac{d}{2}+s-2 k+1+i \nu\right) / 2\right)_{k}\left(\left(\frac{d}{2}+s-2 k+1-i \nu\right) / 2\right)_{k}}, \quad k \neq 0 .
\end{aligned}
$$

As for the massive propagators in the previous section, the $k=0$ term is the traceless and transverse part of the propagator which encodes the exchanged single-particle state, while those for $k>0$ generate purely contact terms in exchange amplitudes.

\subsection{CPWE of spinning exchange diagrams}

In this section we put together the results of the preceding sections to determine CPWEs of tree-level four-point exchange Witten diagrams with fields of arbitrary mass and integer spin on the internal and external legs.

\subsubsection{Natural basis of conformal partial waves in AdS/CFT}

To this end, it is useful to briefly discuss the integral form (2.20) of spinning conformal partial waves in terms of the natural AdS/CFT basis (3.26) of three point conformal structures. Employing this new basis, the spinning conformal partial waves of section 2.2.2 read

$$
\begin{aligned}
& W_{\Delta, s}^{\mathbf{n}, \mathbf{m}}\left(y_{i}\right)+\text { shadow } \\
& =\kappa_{d-\Delta, s} \frac{\gamma_{\tau, s} \bar{\gamma}_{\tau, s}}{\pi^{d / 2}} \int d^{d} y\left[\left[\mathcal{O}_{\Delta_{1}, s_{1}}\left(y_{1}\right) \mathcal{O}_{\Delta_{2}, s_{2}}\left(y_{2}\right) \mathcal{O}_{\Delta, s}(y)\right]\right]^{(\mathbf{n})} \\
& \quad \times\left[\left[\tilde{\mathcal{O}}_{\Delta, s}(y) \mathcal{O}_{\Delta_{3}, s_{3}}\left(y_{3}\right) \mathcal{O}_{\Delta_{4}, s_{4}}\left(y_{4}\right)\right]\right]^{(\mathbf{m})},
\end{aligned}
$$

where for convenience we repeat here the form of the basis elements (3.26)

$$
\begin{aligned}
& {\left[\left[\mathcal{O}_{\Delta_{1}, s_{1}}\left(y_{1}\right) \mathcal{O}_{\Delta_{2}, s_{2}}\left(y_{2}\right) \mathcal{O}_{\Delta_{3}, s_{3}}\left(y_{3}\right)\right]\right]^{(\mathbf{n})}} \\
& \equiv \frac{\mathrm{H}_{1}^{n_{1}} \mathrm{H}_{2}^{n_{2}} \mathrm{H}_{3}^{n_{3}}}{\left(y_{12}\right)^{\delta_{12}}\left(y_{23}\right)^{\delta_{23}}\left(y_{31}\right)^{\delta_{31}}}\left[\prod_{i=1}^{3} 2^{\frac{\delta_{(i+1)(i-1)}}{2}+n_{i}-1} \Gamma\left(\frac{\delta_{(i+1)(i-1)}}{2}+n_{i}\right)\right] \\
& \quad \times\left[\prod_{i=1}^{3} q_{i}^{\frac{1-n_{i}}{2}-\frac{\delta_{(i+1)(i-1)}}{4}} J_{\left(\delta_{(i+1)(i-1)}+2 n_{i}-2\right) / 2}\left(\sqrt{q_{i}}\right)\right] \mathrm{Y}_{1}^{s_{1}-n_{2}-n_{3}} \mathrm{Y}_{2}^{s_{2}-n_{3}-n_{1}} \mathrm{Y}_{3}^{s_{3}-n_{1}-n_{2}}
\end{aligned}
$$

In combination with the basis (3.27) of bulk cubic vertices, once the harmonic function decomposition of a given spinning Witten diagram is known the choice of basis (3.55) of spinning conformal partial waves makes its CPWE follow almost automatically. We demonstrate this explicitly in the following section. 


\subsubsection{Generic spinning exchange diagram}

We consider a generic tree-level four-point exchange of a spin- $s$ field of mass $m^{2}$ between fields of spin $s_{i}$ and mass $m_{i}^{2}$. This is depicted for the s-channel below,

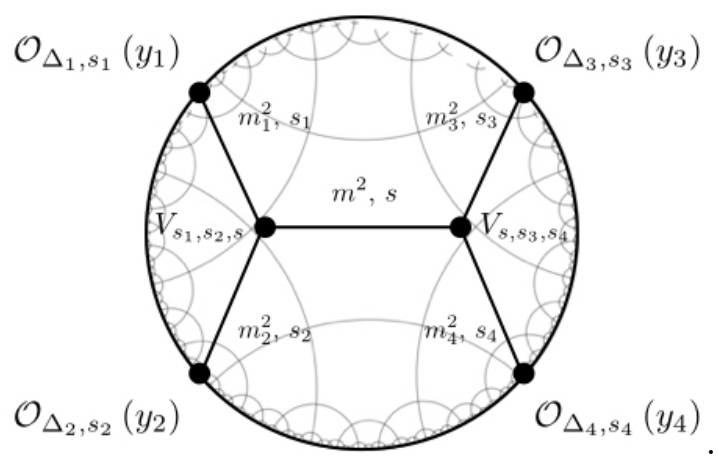

At this level the cubic vertices $V_{s, s_{i}, s_{j}}$ are kinematic, and are not constrained by any consistency condition aside from the necessary requirement of respecting the AdS isometry. Expanded in the natural basis (3.27), they read

$$
V_{s, s_{i}, s_{j}}=\sum_{n_{i}} g_{s_{i}, s_{j}, s}^{n_{i}, n_{j}, n} \mathcal{I}_{s_{i}, s_{j}, s}^{n_{i}, n_{j}, n},
$$

with arbitrary couplings $g_{s_{i}, s_{j}, s}^{n_{i}, n_{j}, n}$.

The harmonic function decomposition follows upon insertion of the massive spin- $s$ bulk-to-bulk propagator (3.40)
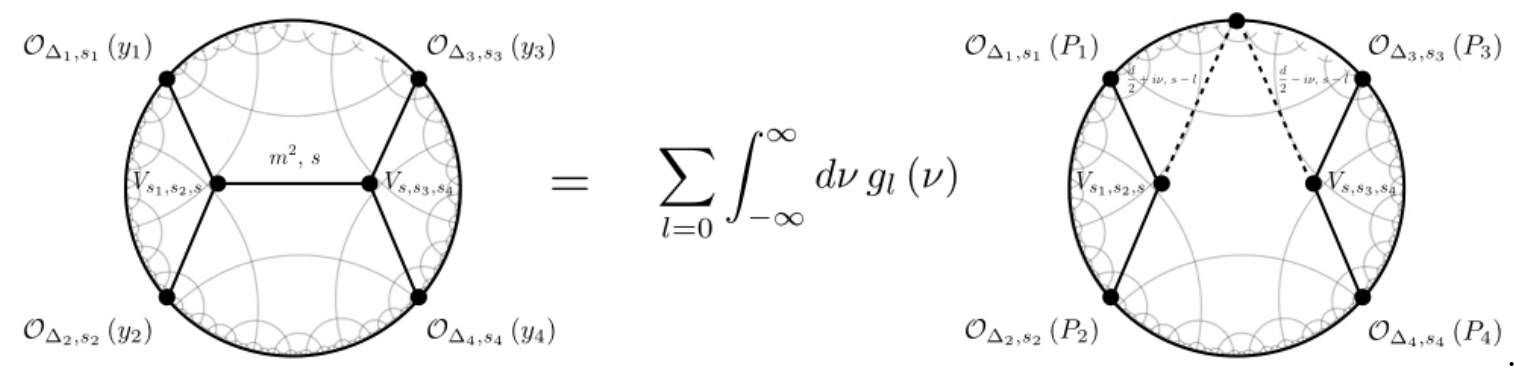

What remains to determine the CPWE is to evaluate the three-point Witten diagrams on the r.h.s., which can be carried out seamlessly by employing the tools developed in section 3.1.

For this generic case we focus on the part of the exchange which encodes the exchanged single-particle state. These are carried by the traceless and transverse part of the bulk-tobulk propagator (3.42), and accordingly we focus on the $l=0$ contribution in the harmonic function decomposition (3.59). The latter is factorised into three-point Witten diagrams 
of the form:

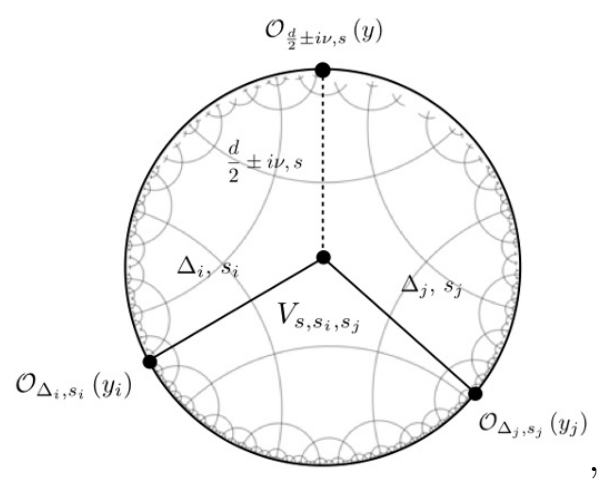

by virtue of the split representation (3.3) of the harmonic function. The amplitudes (3.60) can be straightforwardly given in any basis of three-point conformal structures using the results of section 3.1. However, choosing to expand the couplings (3.58) in the natural AdS/CFT basis (3.27) of cubic vertices gives the following simple and compact form

$$
\begin{aligned}
\mathcal{A}_{\mathbf{s} ; \boldsymbol{\tau}^{ \pm}}\left(y_{i}, y_{j}, y\right) & =\sum_{\mathbf{n}} g_{\mathbf{s}}^{\mathbf{n}} \mathcal{A}_{\mathbf{s} ; \boldsymbol{\tau}^{ \pm}}^{\mathbf{n}}\left(y_{i}, y_{j}, y\right) \\
& =\sum_{\mathbf{n}} g_{\mathbf{s}}^{\mathbf{n}} \mathrm{B}\left(\mathbf{s} ; \mathbf{n} ; \boldsymbol{\tau}^{ \pm}\right)\left[\left[\mathcal{O}_{\Delta_{i}, s_{i}}\left(y_{i}\right) \mathcal{O}_{\Delta_{j}, s_{j}}\left(y_{j}\right) \mathcal{O}_{\frac{d}{2} \pm i \nu, s}(y)\right]\right]^{(\mathbf{n})}
\end{aligned}
$$

where we defined the vectors $\mathbf{s}=\left(s_{i}, s_{j}, s\right), \mathbf{n}=\left(n_{i}, n_{j}, n\right)$ and $\boldsymbol{\tau}^{ \pm}=\left(\tau_{i}, \tau_{j}, \frac{d}{2} \pm i \nu-s\right) .{ }^{29}$

Using the integral representation of the conformal partial waves (3.55), one can then immediately write down the CPWE of the s-channel exchange $(3.57)^{3031}$

$$
\begin{aligned}
\mathcal{A}_{s_{1}, s_{2}\left|s, m^{2}\right| s_{3}, s_{4}}^{\mathrm{s}} & =\int_{-\infty}^{\infty} \frac{d \nu}{\nu^{2}+\left(\frac{d}{2}-\Delta\right)^{2}} \frac{\nu^{2}}{\pi} \mathcal{A}_{\mathbf{s}_{1,2} ; \boldsymbol{\tau}_{1,2}^{+}}\left(y_{1}, y_{2}, y\right) \mathcal{A}_{\mathbf{s}_{3,4} ; \boldsymbol{\tau}_{3,4}^{-}}\left(y_{3}, y_{4}, y\right)+\ldots \\
& =\int_{-\infty}^{\infty} d \nu \sum_{\mathbf{n}, \mathbf{m}} \mathrm{c}_{\mathbf{n}, \mathbf{m}}(\nu) W_{\frac{d}{2}+i \nu, s}^{\mathbf{n}, \mathbf{m}}\left(y_{i}\right)+\ldots
\end{aligned}
$$

with $^{32}$

$$
\mathrm{c}_{\mathbf{n}, \mathbf{m}}(\nu)=-g_{\mathbf{s}_{1,2}}^{\mathbf{n}} g_{\mathbf{s}_{3,4}}^{\mathbf{m}} \frac{\mathrm{B}\left(\mathbf{s}_{12} ; \mathbf{n} ; \boldsymbol{\tau}_{1,2}^{+}\right) \mathrm{B}\left(\mathbf{s}_{34} ; \mathbf{m} ; \boldsymbol{\tau}_{3,4}^{+}\right)}{\nu^{2}+\left(\frac{d}{2}-\Delta\right)^{2}} \frac{2 \pi^{\frac{d}{2}-1} \Gamma(i \nu+1) \nu}{\left(i \nu+s+\frac{d}{2}-1\right) \Gamma\left(i \nu+\frac{d}{2}-1\right)} .
$$

This is the contour integral form (2.14) of the conformal partial wave expansion, reviewed in section 2.2.1. Recall that the functions $\mathbf{c}_{\mathbf{n}, \mathbf{m}}(\nu)$ encode the contribution from spin- $s$

\footnotetext{
${ }^{29}$ In particular, $g_{\mathbf{s}}^{\mathbf{n}}=g_{s_{i}, s_{j}, s}^{n_{i}, n_{j}, n}$ and $\mathcal{A}_{\mathbf{s} ; \boldsymbol{\tau}}^{\mathbf{n}}$ is the amplitude (3.29) with labels $\mathbf{s}=\left(s_{i}, s_{j}, s\right), \mathbf{n}=\left(n_{i}, n_{j}, n\right)$ and $\boldsymbol{\tau}=\left(\tau_{i}, \tau_{j}, \frac{d}{2} \pm i \nu-s\right)$.

${ }^{30}$ Here, $\mathbf{s}_{i, j}=\left(s_{i}, s_{j}, s\right), \boldsymbol{\tau}_{i, j}^{ \pm}=\left(\tau_{i}, \tau_{j}, \frac{d}{2} \pm i \nu-s\right), \mathbf{n}=\left(n_{1}, n_{2}, n\right)$ and $\mathbf{m}=\left(m_{3}, m_{4}, m\right)$.

${ }^{31}$ The ... denote contact terms generated by the $l>0$ contributions in the harmonic function decomposition (3.59).

${ }^{32}$ To obtain this expression we used that

$$
\mathrm{B}\left(\mathbf{s}_{34} ; \mathbf{m} ; \boldsymbol{\tau}_{3,4}^{-}\right)=\frac{i \kappa_{d-i \nu, s} \gamma_{\frac{d}{2}+i \nu-s, s} \bar{\gamma}_{\frac{d}{2}+i \nu-s, s}}{2 \nu \pi^{d / 2} C_{\frac{d}{2}+i \nu, s}} \mathrm{~B}\left(\mathbf{s}_{34} ; \mathbf{m} ; \boldsymbol{\tau}_{3,4}^{+}\right) .
$$


operators: a pole at scaling dimension $\frac{d}{2}+i \nu=\lambda$ in the lower-half $\nu$-plane signifies a contribution from the conformal multiplet $[\lambda, s]$, whose residue gives the corresponding OPE coefficient. Separating out such poles into a function $\mathbf{p}_{\mathbf{n}, \mathbf{m}}(\nu)$,

$$
c_{\mathbf{n}, \mathbf{m}}(\nu)=\bar{c}_{\mathbf{n}, \mathbf{m}}(\nu) \mathbf{p}_{\mathbf{n}, \mathbf{m}}(\nu)
$$

we have

$$
\begin{aligned}
\mathrm{p}_{\mathbf{n}, \mathbf{m}}(\nu)= & \frac{1}{\nu^{2}+\left(\frac{d}{2}-\Delta\right)^{2}} \Gamma\left(\frac{2\left(s_{1}+n-n_{2}\right)+\tau_{1}+\tau_{2}+s-\left(\frac{d}{2}+i \nu\right)}{2}\right) \Gamma\left(\frac{2\left(s_{2}+n-n_{1}\right)+\tau_{1}+\tau_{2}+s-\left(\frac{d}{2}+i \nu\right)}{2}\right) \\
& \times \Gamma\left(\frac{2\left(s_{3}+m-m_{4}\right)+\tau_{3}+\tau_{4}+s-\left(\frac{d}{2}+i \nu\right)}{2}\right) \Gamma\left(\frac{2\left(s_{4}+m-m_{3}\right)+\tau_{3}+\tau_{4}+s-\left(\frac{d}{2}+i \nu\right)}{2}\right) .
\end{aligned}
$$

There are two types of contributions, in accord with the standard lore on CPWEs of Witten diagrams [28, 37, 77, 78, 105, 106, 129-137]:

1. Single-trace: This is the universal contribution to an exchange diagram, corresponding to the exchange of the bulk single-particle state. Accordingly, it is generated by the pole-factor in the traceless and transverse part of the bulk-to-bulk propagator (3.42), which carries the propagating degrees of freedom. This translates into a pole at $\frac{d}{2}+i \nu=\Delta$ in (3.66), which coincides with the scaling dimension of the spin- $s$ single-trace operator $\mathcal{O}_{\Delta, s}$ that is dual to the exchanged spin-s single-particle state of mass $m^{2} R^{2}=\Delta(\Delta-d)-s$ in the bulk.

2. Double-trace: The remaining contributions originate from contact terms, arising from the collision of the two points that are integrated over the entire volume of AdS. This generates 2-particle states in the bulk, which are dual to double-trace operators on the conformal boundary. Accordingly, the corresponding poles are encoded in the factors (3.30) arising from the integration over AdS. In the pole-function (3.66) these are the origin of the two sets of Gamma function poles $(p=0,1,2,3, \ldots)$

1. $\left(\frac{d}{2}+i \nu\right)-s=\tau_{1}+\tau_{2}+2\left(s_{1}+n-n_{2}+p\right), \quad\left(\frac{d}{2}+i \nu\right)-s=\tau_{1}+\tau_{2}+2\left(s_{2}+n-n_{1}+p\right)$

2. $\left(\frac{d}{2}+i \nu\right)-s=\tau_{3}+\tau_{4}+2\left(s_{3}+m-m_{4}+p\right), \quad\left(\frac{d}{2}+i \nu\right)-s=\tau_{3}+\tau_{4}+2\left(s_{4}+m-m_{3}+p\right)$,

corresponding to contributions from the two families $\left[\mathcal{O}_{\Delta_{1}, s_{1}} \mathcal{O}_{\Delta_{2}, s_{2}}\right]_{s}$ and $\left[\mathcal{O}_{\Delta_{3}, s_{3}}\right.$ $\left.\mathcal{O}_{\Delta_{4}, s_{4}}\right]_{s}$ of spin- $s$ double-trace operators, respectively. In the bulk, these correspond to 2-particle states created, respectively, by $\varphi_{s_{1}}$ with $\varphi_{s_{2}}$, and $\varphi_{s_{3}}$ with $\varphi_{s_{4}}$.

Let us briefly comment on the $l>0$ contributions to the harmonic function decomposition (3.59). As explained earlier these are purely contact terms, and likewise generate double-trace contributions $\left[\mathcal{O}_{\Delta_{1}, s_{1}} \mathcal{O}_{\Delta_{2}, s_{2}}\right]_{s-l}$ and $\left[\mathcal{O}_{\Delta_{3}, s_{3}} \mathcal{O}_{\Delta_{4}, s_{4}}\right]_{s-l}$ to the CPWE, but of lower spin $s-l$. 


\subsection{Spinning exchanges in the type A higher-spin gauge theory}

So far our dialogue has not been restricted to any particular theory of spinning fields. In recent years, a lot of interest has been generated in theories of higher-spin gauge fields, owing in part to the conjectured duality [138-143] between higher-spin gauge theories on AdS backgrounds and free CFTs. In this section we apply the tools and results of the preceding sections to compute all four-point exchange Witten diagrams in the simplest higher-spin gauge theory for $d>2$, which is known as the type A minimal higher-spin theory expanded about $\mathrm{AdS}_{d+1}$ [144]. ${ }^{33}$ This theory is conjectured to be dual to the (singlet sector of the) free scalar $O(N)$ model in $d$-dimensions [141, 142].

The spectrum consists of a tower of totally symmetric even spin gauge fields (one for each even spin $s=2,4,6, \ldots)$ and a parity even scalar of mass $m_{0}^{2} R^{2}=-2(d-2)$, which sits in the higher-spin multiplet.

Before moving to the computation of the exchange amplitudes, we first review the result for the metric-like cubic couplings established in [47].

\subsubsection{Off-shell cubic couplings}

The off-shell cubic couplings of the type A minimal higher-spin theory on $\mathrm{AdS}_{d+1}$ were determined in [47], for de Donder gauge. ${ }^{34}$ For tree-level exchanges we only require couplings with a single field - the one that is exchanged — off-shell. For a spin- $s$ field $\varphi_{s}$ in de Donder gauge, its interaction with two on-shell fields of spins $s_{1}$ and $s_{2}$ reads

$$
\begin{aligned}
\mathcal{V}_{s_{1}, s_{2}, s}= & g_{s_{1}, s_{2}, s}\left[1-\frac{1}{2}\left(d-2+\mathcal{Y}_{i} \partial_{\mathcal{Y}_{i}}\right) \partial_{\mathcal{Y}_{3}}^{2} \partial_{U_{3}}^{2}\right] \mathcal{Y}_{1}^{s_{1}} \mathcal{Y}_{2}^{s_{2}} \mathcal{Y}_{3}^{s} \\
& \times \varphi_{s_{1}}\left(X_{1}, U_{1}\right) \varphi_{s_{2}}\left(X_{2}, U_{2}\right) \varphi_{s}\left(X_{3}, U_{3}\right) .
\end{aligned}
$$

The coupling constants $g_{s_{1}, s_{2}, s}$, for canonically normalised kinetic terms, are given by [47]

$$
g_{s_{1}, s_{2}, s}=\frac{1}{\sqrt{N}} \frac{\pi^{\frac{d-3}{4}} 2^{\frac{3 d-1+s_{1}+s_{2}+s}{2}}}{\Gamma\left(d+s_{1}+s_{2}+s-3\right)} \sqrt{\frac{\Gamma\left(s+\frac{d-1}{2}\right)}{\Gamma(s+1)}} \prod_{i=1}^{2} \sqrt{\frac{\Gamma\left(s_{i}+\frac{d-1}{2}\right)}{\Gamma\left(s_{i}+1\right)}} .
$$

The first term in (3.68) is the traceless and transverse part of the vertex, which is non-trivial on-shell. The second term accounts for the off-shell de Donder field $\varphi_{s}$, and accordingly is proportional to its trace.

For the four-point exchange of a spin-s gauge field, we massage the vertices (3.68) into the form

$$
\mathcal{V}_{s_{1}, s_{2}, s}(X)=s ! J_{s \mid s_{1}, s_{2}}\left(X, \partial_{U}\right) \varphi_{s}(X, U)
$$

\footnotetext{
${ }^{33}$ See $[31,37,45,47,145-149]$ for other results on Witten diagrams in higher-spin gauge theories.

${ }^{34}$ Note that although the result (3.68) for the complete cubic couplings was fixed using the holographic duality, it was later verified [53] that the result solves the Noether procedure - i.e. requiring that each cubic coupling is local, the cubic vertices coincide with those that would be obtained without employing holography. The result built upon the covariant classification [99, 150-153] of cubic interactions in $\operatorname{AdS}_{d+1}$.
} 
with the spin- $s$ current $J_{s \mid s_{1}, s_{2}}$ bi-linear in $\varphi_{s_{1}}$ and $\varphi_{s_{2}}$. This is an exercise of integration by parts in ambient space, and gives

$$
J_{s \mid s_{1}, s_{2}}=\left(\sum_{k=0}^{\min \left(s_{1}, s_{2}\right)} \frac{(-2)^{k}}{k !} \frac{\Gamma\left(s_{1}+s_{2}+s+d-3\right)}{\Gamma\left(s_{1}+s_{2}+s+d-3-k\right)} \frac{\Gamma\left(s_{1}+1\right)}{\Gamma\left(s_{1}-k+1\right)} \frac{\Gamma\left(s_{2}+1\right)}{\Gamma\left(s_{2}-k+1\right)} \mathcal{H}_{3}^{k} \overline{\mathcal{Y}}_{1}^{s_{1}-k} \overline{\mathcal{Y}}_{2}^{s_{2}-k} \overline{\mathcal{Y}}_{3}^{s}\right)+\ldots
$$

where the $\ldots$ are terms that constitute the completion with $\varphi_{s}$ off shell, which are reinstated below. For convenience above we defined the contractions

$$
\overline{\mathcal{Y}}_{1}=\mathcal{Y}_{1}, \quad \overline{\mathcal{Y}}_{2}=-\partial_{U_{2}} \cdot \partial_{X_{1}}, \quad \overline{\mathcal{Y}}_{3}=\frac{1}{2} \partial_{U_{3}} \cdot\left(\partial_{X_{1}}-\partial_{X_{2}}\right), \quad \mathcal{H}_{3}=\partial_{U_{1}} \cdot \partial_{U_{2}} .
$$

In its present form, the complete current (3.71) is not exactly conserved. Indeed, recall that for a doubly-traceless Fronsdal field $\varphi_{s}$, higher-spin symmetry at the linearised level only requires that it is conserved up to traces (cf. footnote 27). On the other hand, as emphasised in section 3.2.2, the manifest trace form (3.53) of the bulk-to-bulk propagators requires the use of exactly conserved currents. In appendix B we show the details of how the current (3.71) can be improved such that it satisfies exact conservation. Here we just state that it can be attained by taking on-shell non-trivial part of (3.71) and dressing each term with a differential operator

$$
\mathcal{H}_{3}^{k} \overline{\mathcal{Y}}_{1}^{s_{1}-k} \overline{\mathcal{Y}}_{2}^{s_{2}-k} \overline{\mathcal{Y}}_{3}^{s} \rightarrow\left(\sum_{n=0}^{[s / 2]} \alpha_{n}^{(k)}\left(\partial_{\overline{\mathcal{Y}}_{3}}^{2}\right)^{n}\left(\partial_{U_{3}}^{2}\right)^{n}\right) \mathcal{H}_{3}^{k} \overline{\mathcal{Y}}_{1}^{s_{1}-k} \overline{\mathcal{Y}}_{2}^{s_{2}-k} \overline{\mathcal{Y}}_{3}^{s},
$$

where

$$
\alpha_{n}^{(k)}=\left(\frac{1}{2}\right)^{2 n} \frac{1}{n !} \frac{\Gamma\left(3+k-s_{1}-s_{2}-\frac{d}{2}+n\right)}{\Gamma\left(3+k-s_{1}-s_{2}-\frac{d}{2}\right)} .
$$

\subsubsection{Four-point exchange diagrams}

Consider the four-point exchange of a spin-s gauge field between gauge fields of spin $s_{i}$ in the s-channel. The manifest trace form of the bulk-to-bulk propagator (3.53) gives the harmonic function decomposition
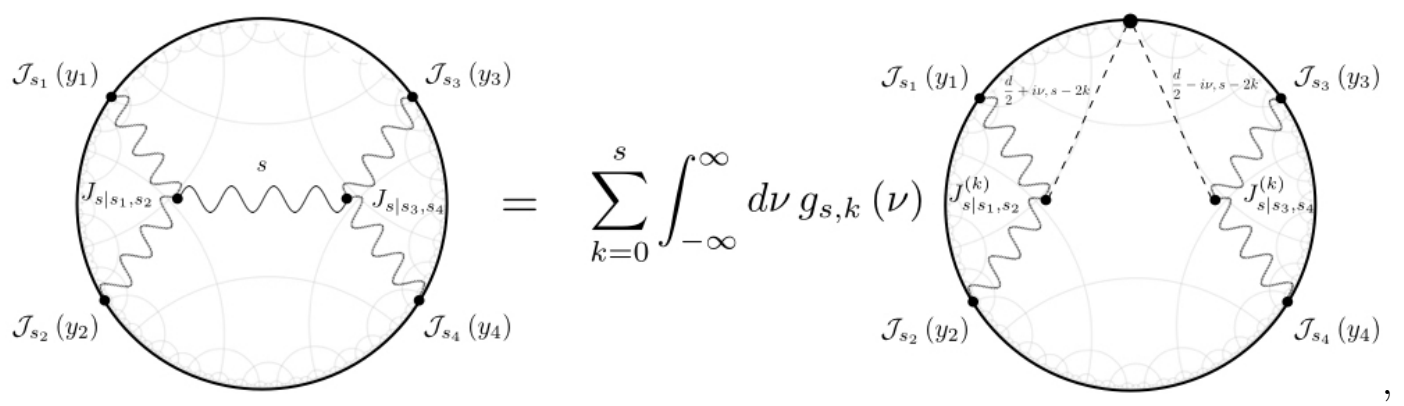

where the operator $\mathcal{J}_{s_{i}}$ is the spin- $s_{i}$ conserved current in the free scalar $O(N)$ model dual to the spin- $s_{i}$ gauge field $\varphi_{s_{i}}$ in the bulk. The notation $J^{(k)}$ denotes the $k$-th trace of the conserved current $J$, which arise from the trace structure of the bulk-to-bulk propagator 
contact terms. The explicit form of $J$ is given in section 3.4.1, while its $k$-th trace is derived in section $\mathrm{C}$.

To determine the CPWE, we therefore need to evaluate three-point Witten diagrams of the form,

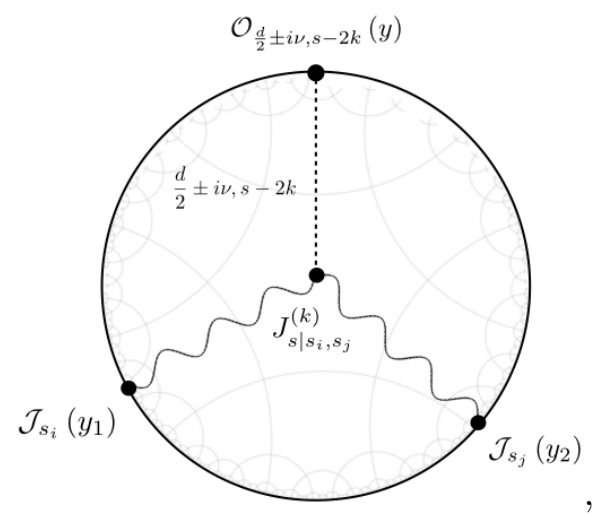

which, employing the tools introduced in section 3.1 entails expressing the cubic couplings in the basis (3.27).

We focus first on the $k=0$ contribution, which encodes the exchanged single-particle state. As we saw for the massive exchanges in section 3.3.2, this is generated by the traceless and transverse part of the bulk-to-bulk propagator (3.53). Accordingly, only the on-shell non-trivial (traceless and transverse) part of the cubic couplings (3.68) contribute, whose explicit form we give here for convenience:

$$
\mathcal{V}_{s_{1}, s_{2}, s}^{T T}(X)=\left.g_{s_{1}, s_{2}, s} \mathcal{Y}_{1}^{s_{1}} \mathcal{Y}_{2}^{s_{2}} \mathcal{Y}_{3}^{s} \varphi_{s_{1}}\left(X_{1}, U_{1}\right) \varphi_{s_{2}}\left(X_{2}, U_{2}\right) \varphi_{s}\left(X_{3}, U_{3}\right)\right|_{X_{i}=X}
$$

Nicely, this is already in the natural AdS/CFT basis (3.27) and the amplitudes (3.76) for $k=0$ can be immediately written down by employing the result (3.29)

$$
\mathcal{A}_{\mathbf{s} ; \boldsymbol{\tau}^{ \pm}}\left(y_{i}, y_{j}, y\right)=g_{s_{i}, s_{j}, s} \mathrm{~B}(\mathbf{s} ; \mathbf{0} ; \boldsymbol{\tau})\left[\left[\mathcal{J}_{s_{i}}\left(y_{i}\right) \mathcal{J}_{s_{j}}\left(y_{j}\right) \mathcal{O}_{\frac{d}{2} \pm i \nu, s}(y)\right]\right]^{\mathbf{0}},
$$

where here $\boldsymbol{\tau}^{ \pm}=\left(d-2, d-2, \frac{d}{2} \pm i \nu-s\right)$ and $\mathbf{n}=(0,0,0)$.

Following the discussion of section 3.3.2 for the generic case, one then obtains that the $k=0$ term in the harmonic function decomposition (3.75) of the exchange diagram yields the following contributions to its CPWE

$$
\mathcal{A}_{s_{1}, s_{2}|s| s_{3}, s_{4}}^{\mathrm{s}}=\int_{-\infty}^{\infty} d \nu \mathrm{c}_{s}(\nu) W_{\frac{d}{2}+i \nu, s}^{\mathbf{0 , 0}}\left(y_{i}\right)+\ldots
$$

with

$$
\mathrm{c}_{s}(\nu)=-g_{s_{1}, s_{2}, s} g_{s_{3}, s_{4}, s} \frac{\mathrm{B}\left(\mathbf{s}_{12} ; \mathbf{0} ; \boldsymbol{\tau}^{+}\right) \mathrm{B}\left(\mathbf{s}_{34} ; \mathbf{0} ; \boldsymbol{\tau}^{+}\right)}{\nu^{2}+\left(s+\frac{d}{2}-2\right)^{2}} \frac{2 \pi^{\frac{d}{2}-1} \Gamma(i \nu+1) \nu}{\left(i \nu+s+\frac{d}{2}-1\right) \Gamma\left(i \nu+\frac{d}{2}-1\right)},
$$

where the pole function (3.66) in this case is given by

$$
\begin{aligned}
\mathrm{p}_{s}(\nu)= & \frac{1}{\nu^{2}+\left(s+\frac{d}{2}-2\right)^{2}} \Gamma\left(\frac{2\left(s_{1}+d-2\right)+s-\left(\frac{d}{2}+i \nu\right)}{2}\right) \Gamma\left(\frac{2\left(s_{2}+d-2\right)+s-\left(\frac{d}{2}+i \nu\right)}{2}\right) \\
& \times \Gamma\left(\frac{2\left(s_{3}+d-2\right)+s-\left(\frac{d}{2}+i \nu\right)}{2}\right) \Gamma\left(\frac{2\left(s_{4}+d-2\right)+s-\left(\frac{d}{2}+i \nu\right)}{2}\right) .
\end{aligned}
$$

In the following we discuss in detail the particular contributions. 
Single-trace. In line with the discussion of the generic case in section 3.3.2, the pole factor in the traceless and transverse part of the bulk-to-bulk propagator (3.53) generates a pole in (3.81) at $\frac{d}{2}+i \nu=s+d-2$, which is the scaling dimension of the dual spin- $s$ conserved current $\mathcal{J}_{s}$ in the free scalar $O(N)$ model.

Furthermore, notice that for $\frac{d}{2}+i \nu=s+d-2$ the three-point conformal structure generated by the $k=0$ amplitude (3.78) coincides with the three-point conserved structure (2.28) in free scalar theories. The corresponding spin- $s$ conformal partial wave thus coincides with the conserved conformal partial wave $\mathcal{W}_{s_{1}, s_{2}|s| s_{3}, s_{4}}^{0,0}$ in the set (2.25), which represents the contribution from the conserved operator $\mathcal{J}_{s}$ to the four-point function $\left\langle\mathcal{J}_{s_{1}} \mathcal{J}_{s_{2}} \mathcal{J}_{s_{3}} \mathcal{J}_{s_{4}}\right\rangle$ in free scalar theories.

With the result (2.29) of all single-trace conserved current OPE coefficients in free scalar theories, in this case we can confirm the standard expectation that the single-trace contribution to an exchange Witten diagram coincides with the contribution from the same single-trace operator in the CPWE of the dual CFT four-point function, when expanded in the same channel. Indeed, using that $[47,53]^{35}$

$$
g_{s_{i}, s_{j}, s} \frac{\mathrm{B}\left(\mathbf{s}_{i j} ; \mathbf{0} ; d-2, d-2, d-2\right)}{\sqrt{C_{s_{i}+d-2, s_{i}} C_{s_{j}+d-2, s_{j}} C_{s+d-2, s}}}=\mathrm{c}_{\mathcal{J}_{s_{i}} \mathcal{J}_{s_{j}} \mathcal{J}_{s}},
$$

we have (closing the contour in the lower-half $\nu$-plane)

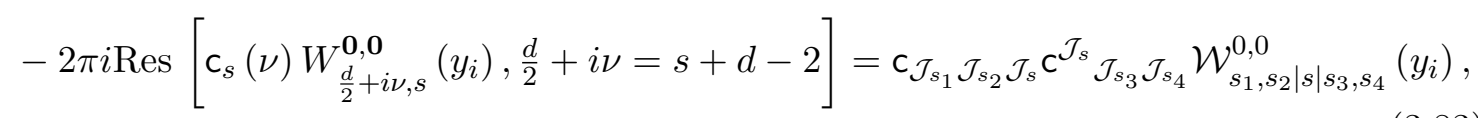

as expected.

Double-trace. As usual these originate from contact terms in the $k=0$ part of the exchange. In this case the two sets of Gamma function poles in (3.81)

$$
\begin{aligned}
& \text { 1. }\left(\frac{d}{2}+i \nu\right)-s=2(d-2)+2\left(p+s_{1}\right), \quad\left(\frac{d}{2}+i \nu\right)-s=2(d-2)+2\left(p+s_{2}\right), \\
& \text { 2. }\left(\frac{d}{2}+i \nu\right)-s=2(d-2)+2\left(p+s_{3}\right), \quad\left(\frac{d}{2}+i \nu\right)-s=2(d-2)+2\left(p+s_{4}\right),
\end{aligned}
$$

with $p=0,1,2,3, \ldots$, correspond to contributions from the two families $\left[\mathcal{J}_{s_{1}} \mathcal{J}_{s_{2}}\right]_{s}$ and $\left[\mathcal{J}_{s_{3}} \mathcal{J}_{s_{4}}\right]_{s}$ of spin- $s$ double-trace operators built from single-trace conserved currents.

$\boldsymbol{k}>\mathbf{0}$ contributions. Similarly, being contact, the $k>0$ contributions to the CPWE of the exchange (3.75) are from double-trace operators $\left[\mathcal{J}_{s_{1}} \mathcal{J}_{s_{2}}\right]_{s-2 k}$ and $\left[\mathcal{J}_{s_{3}} \mathcal{J}_{s_{4}}\right]_{s-2 k}$ of lower spin $s-2 k$. The result for the corresponding three-point Witten diagrams (3.76) for $k>0$ is a lot more involved, as it requires to compute the $k$-th trace of the currents $J_{s \mid s_{i}, s_{j}}^{(k)}$. We show explicitly how the latter are computed in section $\mathrm{C}$, from which the results for the corresponding three-point Witten diagrams (3.76) are given immediately from the results of section 3.1. This gives the CPWE of the $k>0$ contributions via (3.75).

\footnotetext{
${ }^{35}$ Here we divide by the normalisation of the bulk-to-boundary propagators (3.12) to give unit normalisation to the dual single-trace operator two point functions (3.13).
} 
We point out that there are simplifications for particular combinations of the external spins, such as for a single spinning external field (e.g. $\left.s_{1}-0-0-0\right)$ and also for a single spinning external field either side of the exchange (e.g. $s_{1}-0-s_{3}-0$ in the s-channel), where the threepoint bulk integrals for $k>0$ are of the same type as in the $k=0$ case.

Note that in $\mathrm{AdS}_{4}$ (the case relevant for the duality with the critical $O(N)$ model) the current-type interactions $J_{s \mid s_{i}, s_{j}}$ are traceless via improvements, and so the $k>0$ contributions are vanishing.

\section{Acknowledgments}

C. S. and M. T. are grateful to M. Henneaux for useful discussions, and also A. Castro, E. Llabrés and F. G. Rejón-Barrera in the context of geodesic Witten diagrams. C. S. also thanks D. Francia for useful correspondence. The research of M. T. is partially supported by the Fund for Scientific Research-FNRS Belgium, grant FC 6369 and by the Russian Science Foundation grant 14-42-00047 in association with Lebedev Physical Institute.

\section{A Conventions, notations and ambient space}

In this work we employ the same conventions as in [47], which we very briefly review here for completeness. For more details on the ambient space formalism, see for instance [99, $154,155]$.

The ambient formalism is an indispensable framework for computations in $\operatorname{AdS}_{d+1}$ space. In this context, the latter is viewed as a hyperboloid embedded in an ambient $(d+2)$-dimensional Minkowski space

$$
X^{2}+R^{2}=0, \quad X^{0}>0,
$$

where $R$ is the AdS radius. In ambient light-cone coordinates $\left(X^{+}, X^{-}, X^{i}\right)$ with $X^{2}=$ $-X^{+} X^{-}+\delta_{i j} X^{i} X^{j}$, the solution of the constraints (A.1) in the Poincaré co-ordinates $x^{\mu}=\left(z, y^{i}\right)$ is given by

$$
X^{A}=\frac{R}{z}\left(1, z^{2}+y^{2}, y^{i}\right) .
$$

Bulk fields. In order to obtain a one-to-one correspondence between fields on AdS and those living in the higher-dimensional flat ambient space, one imposes constraints with defining the ambient space extensions of the $\mathrm{AdS}_{d+1}$ fields [108]. Such restrictions are usually given as homogeneity and tangentiality constraints. Employing a generating function formalism with intrinsic and ambient auxiliary vectors $u^{\mu}$ and $U^{A}$, a symmetric rank- $s$ tensor $\varphi_{s}(x, u)$ intrinsic to the AdS manifold is represented in ambient space by

$$
\varphi_{s}(x, u)=\frac{1}{s !} \varphi_{\mu_{1} \ldots \mu_{s}}(x) u^{\mu_{1}} \ldots u^{\mu_{s}} \quad \rightarrow \quad \varphi_{s}(X, U)=\frac{1}{s !} \varphi_{A_{1} \ldots A_{s}}(X) U^{A_{1}} \ldots U^{A_{s}} .
$$

subject to the following homogeneity and tangentiality conditions

$$
\left(X \cdot \partial_{X}-\Delta\right) \varphi_{s}(X, U)=0, \quad\left(X \cdot \partial_{U}\right) \varphi_{s}(X, U)=0 .
$$


Nicely, the conditions (A.4) imply that on-shell

$$
\partial_{X}^{2} \varphi_{A_{1} \ldots A_{s}}=0
$$

for the ambient representative of the AdS field $\varphi_{s}$ of mass $m^{2} R^{2}=\Delta(\Delta-d)-s$.

Let us stress that in imposing tangentiality and homogeneity conditions (A.4) one is implicitly extending the AdS field to the full ambient space, where $X^{2}$ plays the role of the radial coordinate. This formalism is different from the manifestly intrinsic formalism (for instance used in [40]) where one never moves away from the AdS manifold $X^{2}=-R^{2}$.

The ambient representative of the AdS covariant derivative $\nabla_{\mu}$ takes the simple form

$$
\nabla_{A}=\mathcal{P}_{A}^{B} \frac{\partial}{\partial X^{B}}
$$

and acts via

$$
\nabla=\mathcal{P} \circ \partial \circ \mathcal{P}
$$

Boundary fields. The boundary of $\mathrm{AdS}_{d+1}$ is identified with the null rays

$$
P^{2}=0, \quad P \sim \lambda P, \quad \lambda \neq 0,
$$

where $P$ gives the ambient space embedding of the CFT coordinate $y^{i}$. It is convenient to introduce the boundary analog of the auxiliary variables $U^{A}$, which we refer to as $Z_{A}(y)$ and extend to ambient space the null CFT auxiliary variable $z^{i}$. Working in light cone coordinates $P^{A}=\left(P^{+}, P^{-}, P^{i}\right)$, with the gauge choice $P^{+}=1$ one has

$$
P^{A}(y)=\left(1, y^{2}, y^{i}\right) \quad \text { and } \quad Z^{A}(y)=\left(0,2 y \cdot z, z^{i}\right)
$$

A symmetric rank-s boundary operator $O_{\Delta, s}$ of scaling dimension $\Delta$ is represented by: ${ }^{36}$

$$
O_{\Delta, s}(y, z)=\frac{1}{s !} O_{\mu_{1} \ldots \mu_{s}}(y) z^{\mu_{1}} \cdots z^{\mu_{s}} \quad \rightarrow \quad \mathcal{O}_{\Delta, s}(P, Z)=\frac{1}{s !} \mathcal{O}_{A_{1} \ldots A_{s}}(P) Z^{A_{1}} \cdots Z^{A_{s}},
$$

where

$$
\left(P \cdot \partial_{P}-\Delta\right) O_{\Delta, s}(P, Z)=0, \quad\left(P \cdot \partial_{Z}\right) \mathcal{O}_{\Delta, s}(P, Z)=0
$$

and, being restricted to the null cone (A.8), there is an extra redundancy

$$
\begin{gathered}
O_{A_{1} \ldots A_{s}}(P) \rightarrow O_{A_{1} \ldots A_{s}}(P)+P_{\left(A_{1}\right.} \Lambda_{\left.A_{2} \ldots A_{s}\right)}, \\
P^{A_{1}} \Lambda_{A_{1} \ldots A_{s-1}}=0, \quad \Lambda_{A_{1} \ldots A_{s-1}}(\lambda P)=\lambda^{-(\Delta+1)} \Lambda_{A_{1} \ldots A_{s-1}}(P), \quad \eta^{A_{1} A_{2}} \Lambda_{A_{1} \ldots A_{s-1}}=0 .
\end{gathered}
$$

\footnotetext{
${ }^{36}$ Note that here $z$ denotes the auxiliary vector $z^{i}$ and should not be confused with the radial Poincaré co-ordinate in (A.2).
} 


\section{B The improved current}

In this appendix we detail the improvement of the higher-spin currents (3.71) to make them exactly conserved. We begin with the traceless and transverse part of the current,

$$
J_{s_{3} \mid s_{1}, s_{2}}^{T T}=\sum_{k=0}^{\min \left(s_{1}, s_{2}\right)} \frac{(-2)^{k}}{k !} \frac{\Gamma\left(s_{1}+s_{2}+s_{3}+d-3\right)}{\Gamma\left(s_{1}+s_{2}+s_{3}+d-3-k\right)} \frac{\Gamma\left(s_{1}+1\right)}{\Gamma\left(s_{1}-k+1\right)} \frac{\Gamma\left(s_{2}+1\right)}{\Gamma\left(s_{2}-k+1\right)} \mathcal{H}_{3}^{k} \overline{\mathcal{Y}}_{1}^{s_{1}-k} \overline{\mathcal{Y}}_{2}^{s_{2}-k} \overline{\mathcal{Y}}_{3}^{s_{3}} .
$$

On-shell, each monomial in the above is conserved. One can therefore study the structure of the required improvements with $\varphi_{3}$ off-shell for a given monomial

$$
f_{s_{1}, s_{2}, s_{3}}^{(k)}=\mathcal{H}_{3}^{k} \overline{\mathcal{Y}}_{1}^{s_{1}-k} \overline{\mathcal{Y}}_{2}^{s_{2}-k} \overline{\mathcal{Y}}_{3}^{s_{3}} .
$$

The combination of different monomials in (B.1) above is necessary to achieve on-shell gauge invariance with respect to $\varphi_{1}$ and $\varphi_{2}$ which can be easily verified explicitly (see e.g. [99]).

In order to proceed to find the conserved improvement, the doubly-traceless condition on $\varphi_{3}$ together with the traceless condition on the corresponding gauge parameter needs to be dropped. Not doing so would only recover a current whose traceless part is conserved. We hence consider the following ansatz for the improvement, dressing each monomial (B.2) with trace operators

$$
F_{s_{1}, s_{2}, s_{3}}^{(k)}=\left(\sum_{n=0}^{\left[s_{3} / 2\right]} \alpha_{n}^{(k)}\left(\partial_{\overline{\mathcal{Y}}_{3}}^{2}\right)^{n}\left(\partial_{U_{3}}^{2}\right)^{n}\right) f_{s_{1}, s_{2}, s_{3}}^{(k)}
$$

where the derivative with respect to $\overline{\mathcal{Y}}_{3}$ accounts for the fact that taking the trace lowers the spin. The coefficients $\alpha_{n}^{(k)}$ in the ansatz (B.3) are fixed by requiring gauge invariance of the vertex with $\varphi_{3}$ off-shell and with traceless gauge parameter $\xi_{3}$

$$
\int d X\left(U_{3} \cdot \nabla_{3} \xi_{3}\right) F_{s_{1}, s_{2}, s_{3}}^{(k)} \varphi_{1} \varphi_{2}=0
$$

In the above the fields $\varphi_{1}$ and $\varphi_{2}$ are on-shell, while the integral sign (which in the following will be omitted for ease of notation) implies that the above identity holds modulo total derivatives.

Employing the explicit form of the gradient operator:

$$
U_{3} \cdot \nabla_{3}=U_{3} \cdot \partial_{X_{3}}-\frac{U_{3} \cdot X_{3}}{X_{3}^{2}}\left(X_{3} \cdot \partial_{X_{3}}-U_{3} \cdot \partial_{U_{3}}\right),
$$

we arrive to the following conservation condition:

$$
\sum_{n=0}^{\infty}\left[2(n+1) \alpha_{n+1}^{(k)}-\frac{1}{4} \alpha_{n}^{(k)}\left[d+2\left(s_{1}+s_{2}-k-n\right)-4\right]\right]\left(\partial_{\mathcal{Y}_{3}}^{2}\right)^{n} f_{s_{1}, s_{2}, s_{3}}^{(k)}\left(\partial_{U_{3}}^{2}\right)^{n} \partial_{U_{3}} \cdot \partial_{X_{3}}=0,
$$

which leads to the solution for $\alpha_{n}^{(k)}$ in the form

$$
\alpha_{n}^{(k)}=\left(\frac{1}{2}\right)^{2 n} \frac{1}{n !} \frac{\Gamma\left(3+k-s_{1}-s_{2}-\frac{d}{2}+n\right)}{\Gamma\left(3+k-s_{1}-s_{2}-\frac{d}{2}\right)} .
$$




\section{Trace of the currents}

In order to evaluate the current exchange (3.75) with the manifest trace form of the propagator (3.53), we are required to compute the $n$-th trace of the exactly conserved current derived in the previous section. The process can be simplified by noting that in the present context the traces are contracted with harmonic functions, where we encounter terms of the form

$$
\left.\left(\partial_{\overline{\mathcal{Y}}_{3}}^{2}\right)^{n} f_{s_{1}, s_{2}, s_{3}}^{(k)}\left(\partial_{U_{3}}^{2}\right)^{n}\left(u_{3}^{2}\right)^{q} \Omega_{\nu, s_{3}-2 q}\right|_{U_{3}=0},
$$

where $u_{3}^{2}$ is the intrinsic symmetrised metric tensor written in generating function form, which can be re-expressed in the ambient formalism as

$$
u_{3}^{2}=U_{3}^{2}-\frac{U_{3} \cdot X_{3} U_{3} \cdot X_{3}}{X_{3}^{2}} .
$$

To evaluate the trace one commutes the $U_{3}$ contained in the $\left(u_{3}^{2}\right)^{q}$ to the far left hand side, where the condition $U_{3}=0$ can be applied. We first commute the $u_{3}^{2}$ past the $\partial_{U_{3}}^{2}$, which, employing the tracelessness of the harmonic functions, reads

$$
\left(\partial_{U_{3}}^{2}\right)^{n}\left(u_{3}^{2}\right)^{q} \Omega_{\nu, s_{3}-2 q}=A_{n}^{q}\left(u_{3}^{2}\right)^{q-n} \Omega_{\nu, s_{3}-2 q}, \quad A_{n}^{q}=2^{2 n} \frac{\Gamma(n-q)}{\Gamma(-q)} \frac{\Gamma\left(-\frac{d}{2}+n+q-s_{3}+\frac{1}{2}\right)}{\Gamma\left(-\frac{d}{2}+q-s_{3}+\frac{1}{2}\right)} .
$$

What remains is to evaluate terms of the form

$$
\left.\left(\partial_{\overline{\mathcal{Y}}_{3}}^{2}\right)^{n} f_{s_{1}, s_{2}, s_{3}}^{(k)}\left(u_{3}^{2}\right)^{q-n} \Omega_{\nu, s_{3}-2 q}\right|_{U_{3}=0} .
$$

To this end, it is useful split $\overline{\mathcal{Y}}_{3}$ as ${ }^{37}$

$$
\overline{\mathcal{Y}}_{3}=\frac{1}{2}\left(V_{31}-V_{32}\right), \quad V_{31}=\partial_{U_{3}} \cdot \partial_{X_{1}}, \quad V_{31}=\partial_{U_{3}} \cdot \partial_{X_{2}},
$$

and express any function of $\overline{\mathcal{Y}}_{3}$ instead in terms of $V_{31}$ and $V_{21}$. In this way, the action of some operator $g\left(\overline{\mathcal{Y}}_{3}\right)$ on $u_{3}$ can be expressed in the form

$$
\left.g\left(\overline{\mathcal{Y}}_{3}\right) u_{3}^{A}\right|_{U_{3}=0}=\left(\partial_{X_{1}}^{A} \partial_{V_{31}}+\partial_{X_{2}}^{A} \partial_{V_{32}}\right) g\left(V_{31}, V_{32}\right),
$$

from which follows the general formula

$$
\begin{aligned}
\left.g\left(\overline{\mathcal{Y}}_{3}\right) u_{3}^{2}\right|_{U_{3}=0} & =\left\{2\left[\partial_{X_{1}} \cdot \partial_{X_{2}}+X_{1} \cdot \partial_{X_{1}} X_{2} \cdot \partial_{X_{2}}\right] \partial_{V_{31}} \partial_{V_{32}}\right. \\
& \left.+X_{1} \cdot \partial_{X_{1}}\left(X_{1} \cdot \partial_{X_{1}}-1\right) \partial_{V_{31}}^{2}+X_{2} \cdot \partial_{X_{2}}\left(X_{2} \cdot \partial_{X_{2}}-1\right) \partial_{V_{32}}^{2}\right\} g\left(V_{31}, V_{32}\right)
\end{aligned}
$$

which can be iteratively applied to evaluate the traces in (C.4). Now, since each current in the exchange is to be integrated over AdS, we can evaluate the above terms up to integrations by parts using

$$
\begin{aligned}
& X_{1} \cdot \partial_{X_{1}}=-\left(d-2+\overline{\mathcal{Y}}_{1} \partial_{\overline{\mathcal{Y}}_{1}}+\overline{\mathcal{Y}}_{2} \partial_{\overline{\mathcal{Y}}_{2}}+V_{31} \partial_{V_{31}}+Q_{3} \partial_{Q_{3}}\right), \\
& X_{2} \cdot \partial_{X_{2}}=-\left(d-2+\overline{\mathcal{Y}}_{1} \partial_{\overline{\mathcal{Y}}_{1}}+\overline{\mathcal{Y}}_{2} \partial_{\overline{\mathcal{Y}}_{2}}+V_{32} \partial_{V_{32}}+Q_{3} \partial_{Q_{3}}\right),
\end{aligned}
$$

\footnotetext{
${ }^{37}$ Note that in fact $V_{31}=\mathcal{Y}_{3}$, but for ease of notation in this section we employ the labelling $V_{31}$.
} 
where

$$
Q_{3}=-\frac{1}{2}\left(X_{1} \cdot \partial_{X_{1}}+X_{1} \cdot \partial_{X_{1}}+\Delta_{3}+d\right)\left(X_{1} \cdot \partial_{X_{1}}+X_{1} \cdot \partial_{X_{1}}-\Delta_{3}\right),
$$

and $\Delta_{3}=\frac{d}{2} \pm i \nu$

After evaluating the action of the above operators one can integrate by parts to obtain an expression for the final form of the trace terms (C.1) in the form

$$
J_{s_{3} \mid s_{1}, s_{2}}^{(k)} \cdot \Pi_{\frac{d}{2} \pm i \nu, s_{3}-2 k}=\sum_{m=0}^{\min \left(s_{1}, s_{2}\right)} \beta_{s_{3} \mid s_{1}, s_{2}}^{k, m} \mathcal{H}_{3}^{m} \mathcal{Y}_{1}^{s_{1}-m} \mathcal{Y}_{2}^{s_{2}-m} \mathcal{Y}_{3}^{s_{3}-2 k},
$$

where, via integration by parts, we replaced $\overline{\mathcal{Y}}_{2} \rightarrow e^{\lambda \mathcal{H}_{3} \partial_{\mathcal{Y}_{1}} \partial_{\mathcal{Y}_{2}}}, V_{31} \rightarrow \mathcal{Y}_{3}$ and $V_{32} \rightarrow$ $-\mathcal{Y}_{3}+\partial_{U_{3}} \cdot \partial_{X_{3}}=-\mathcal{Y}_{3}$, where in the latter equality we used that the harmonic function is divergenceless. See $[47,99]$ for (in-context) reviews of integration by parts in the ambient space formalism, where in particular the parameter $\lambda$ and its use is defined.

Equation (3.18) gives the three-point amplitude (3.76) generated by (C.11).

\section{Seed bulk integrals}

Our approach to evaluate spinning three-point Witten diagrams is underpinned by their differential relationship (3.11) with basic seed diagrams with external scalars [100]. The latter is the basic ingredient from which our results are generated, which we briefly review here.

It is useful to employ the Schwinger-parameterised form for the propagator $[156,157]$

$$
K_{\Delta}(X ; P)=\frac{C_{\Delta, 0}}{\Gamma(\Delta)} \int_{0}^{\infty} \frac{d t}{t} t^{\Delta} e^{2 t P \cdot X},
$$

which results in

$$
A_{0,0,0 ; \tau_{1}, \tau_{2}, \tau_{3}}^{0,0,0}\left(P_{1}, P_{2}, P_{3}\right)=\int_{0}^{\infty} \prod_{i=1}^{3}\left(\frac{C_{\Delta_{i}, 0}}{\Gamma\left(\Delta_{i}\right)} \frac{d t_{i}}{t_{i}} t^{\Delta_{i}}\right) \int_{\text {AdS }} d X e^{2\left(t_{1} P_{1}+t_{2} P_{2}+t_{3} P_{3}\right) \cdot X}
$$

The integration over AdS is then straightforward to perform, and yields (see e.g. box 5.2 in $[155])$

$$
\begin{aligned}
\int_{0}^{\infty} \prod_{i=1}^{3}\left(\frac{d t_{i}}{t_{i}} t^{\Delta_{i}}\right) & \int_{\mathrm{AdS}} d X e^{2\left(t_{1} P_{1}+t_{2} P_{2}+t_{3} P_{3}\right) \cdot X} \\
& =\pi^{\frac{d}{2}} \Gamma\left(\frac{-d+\sum_{i=1}^{3} \Delta_{i}}{2}\right) \int_{0}^{\infty} \prod_{i=1}^{3}\left(\frac{d t_{i}}{t_{i}} t_{i}^{\Delta_{i}}\right) e^{\left(-t_{1} t_{2} P_{12}-t_{1} t_{3} P_{13}-t_{2} t_{3} P_{23}\right)}
\end{aligned}
$$

where $P_{i j}=-2 P_{i} \cdot P_{j}$. Through the change of variables,

$$
t_{1}=\sqrt{\frac{m_{2} m_{3}}{m_{1}}}, \quad t_{2}=\sqrt{\frac{m_{1} m_{3}}{m_{2}}}, \quad t_{3}=\sqrt{\frac{m_{1} m_{2}}{m_{3}}},
$$


we then obtain the final result:

$$
\begin{aligned}
& A_{0,0,0 ; \tau_{1}, \tau_{2}, \tau_{3}}^{0,0,0}\left(P_{1}, P_{2}, P_{3}\right) \\
& =\frac{\pi^{\frac{d}{2}}}{2} \Gamma\left(\frac{-d+\sum_{i=1}^{3} \Delta_{i}}{2}\right)\left(\prod_{i=1}^{3} \frac{C_{\Delta_{i}, 0}}{\Gamma\left(\Delta_{i}\right)}\right) \\
& \times \int_{0}^{\infty} \prod_{i=1}^{3}\left(\frac{d m_{i}}{m_{i}} m_{i}^{\delta_{(i+1)(i-1)} / 2}\right) \exp \left(-m_{i} P_{(i+1)(i-1)}\right),
\end{aligned}
$$

where $i \cong i+3$ and

$$
\delta_{i(i+1)}=\frac{\Delta_{i}+\Delta_{(i+1)}-\Delta_{(i-1)}}{2},
$$

The standard three-point conformal structure for scalar operators is obtained from (D.5) using the integral representation of the Gamma function

$$
A_{0,0,0 ; \tau_{1}, \tau_{2}, \tau_{3}}^{0,0,0}\left(P_{1}, P_{2}, P_{3}\right)=\mathrm{C}\left(\Delta_{1}, \Delta_{2}, \Delta_{3} ; 0\right) \frac{1}{P_{13}^{\frac{\Delta_{1}+\Delta_{3}-\Delta_{2}}{2}} P_{23}^{\frac{\Delta_{2}+\Delta_{3}-\Delta_{1}}{2}} P_{12}^{\frac{\Delta_{1}+\Delta_{2}-\Delta_{3}}{2}}},
$$

where explicitly

$$
\begin{aligned}
\mathrm{C}\left(\Delta_{1}, \Delta_{2}, \Delta_{3} ; 0\right) \\
=\frac{1}{2} \pi^{\frac{d}{2}} \Gamma\left(\frac{-d+\sum_{i=1}^{3} \Delta_{i}}{2}\right) C_{\Delta_{1}, 0} C_{\Delta_{2}, 0} C_{\Delta_{3}, 0} \\
\quad \times \frac{\Gamma\left(\frac{\Delta_{1}+\Delta_{2}-\Delta_{3}}{2}\right) \Gamma\left(\frac{\Delta_{1}+\Delta_{3}-\Delta_{2}}{2}\right) \Gamma\left(\frac{\Delta_{2}+\Delta_{3}-\Delta_{1}}{2}\right)}{\Gamma\left(\Delta_{1}\right) \Gamma\left(\Delta_{2}\right) \Gamma\left(\Delta_{3}\right)} .
\end{aligned}
$$

Open Access. This article is distributed under the terms of the Creative Commons Attribution License (CC-BY 4.0), which permits any use, distribution and reproduction in any medium, provided the original author(s) and source are credited.

\section{References}

[1] S. Ferrara, A.F. Grillo, G. Parisi and R. Gatto, The shadow operator formalism for conformal algebra. vacuum expectation values and operator products, Lett. Nuovo Cim. 4 (1972) 115.

[2] G. Mack and I.T. Todorov, Conformal-invariant green functions without ultraviolet divergences, Phys. Rev. D 8 (1973) 1764 [INSPIRE].

[3] S. Ferrara, A.F. Grillo and R. Gatto, Tensor representations of conformal algebra and conformally covariant operator product expansion, Annals Phys. 76 (1973) 161 [INSPIRE].

[4] S. Ferrara, A.F. Grillo, G. Parisi and R. Gatto, Covariant expansion of the conformal four-point function, Nucl. Phys. B 49 (1972) 77 [Erratum ibid. B 53 (1973) 643] [INSPIRE].

[5] A.M. Polyakov, Nonhamiltonian approach to conformal quantum field theory, Zh. Eksp. Teor. Fiz. 66 (1974) 23 [INSPIRE].

[6] S. Ferrara, A.F. Grillo, R. Gatto and G. Parisi, Analyticity properties and asymptotic expansions of conformal covariant green's functions, Nuovo Cim. A 19 (1974) 667 [INSPIRE]. 
[7] A.A. Belavin, A.M. Polyakov and A.B. Zamolodchikov, Infinite Conformal Symmetry in Two-Dimensional Quantum Field Theory, Nucl. Phys. B 241 (1984) 333 [inSPIRE].

[8] F.A. Dolan and H. Osborn, Conformal four point functions and the operator product expansion, Nucl. Phys. B 599 (2001) 459 [hep-th/0011040] [INSPIRE].

[9] F.A. Dolan and H. Osborn, Conformal partial waves and the operator product expansion, Nucl. Phys. B 678 (2004) 491 [hep-th/0309180] [INSPIRE].

[10] R. Rattazzi, V.S. Rychkov, E. Tonni and A. Vichi, Bounding scalar operator dimensions in $4 D$ CFT, JHEP 12 (2008) 031 [arXiv:0807.0004] [INSPIRE].

[11] V.S. Rychkov and A. Vichi, Universal Constraints on Conformal Operator Dimensions, Phys. Rev. D 80 (2009) 045006 [arXiv:0905.2211] [INSPIRE].

[12] F. Caracciolo and V.S. Rychkov, Rigorous Limits on the Interaction Strength in Quantum Field Theory, Phys. Rev. D 81 (2010) 085037 [arXiv: 0912. 2726] [inSPIRE].

[13] F.A. Dolan and H. Osborn, Conformal Partial Waves: Further Mathematical Results, arXiv:1108.6194 [INSPIRE].

[14] M.F. Paulos, JuliBootS: a hands-on guide to the conformal bootstrap, arXiv:1412.4127 [INSPIRE].

[15] D. Simmons-Duffin, A Semidefinite Program Solver for the Conformal Bootstrap, JHEP 06 (2015) 174 [arXiv: 1502.02033] [INSPIRE].

[16] S. El-Showk, M.F. Paulos, D. Poland, S. Rychkov, D. Simmons-Duffin and A. Vichi, Solving the 3D Ising Model with the Conformal Bootstrap, Phys. Rev. D 86 (2012) 025022 [arXiv:1203.6064] [INSPIRE].

[17] F. Kos, D. Poland, D. Simmons-Duffin and A. Vichi, Precision Islands in the Ising and $O(N)$ Models, JHEP 08 (2016) 036 [arXiv: 1603.04436] [inSPIRE].

[18] A.L. Fitzpatrick, J. Kaplan, D. Poland and D. Simmons-Duffin, The Analytic Bootstrap and AdS Superhorizon Locality, JHEP 12 (2013) 004 [arXiv:1212.3616] [INSPIRE].

[19] Z. Komargodski and A. Zhiboedov, Convexity and Liberation at Large Spin, JHEP 11 (2013) 140 [arXiv: 1212.4103] [inSPIRE].

[20] L.F. Alday and A. Zhiboedov, Conformal Bootstrap With Slightly Broken Higher Spin Symmetry, JHEP 06 (2016) 091 [arXiv: 1506. 04659] [INSPIRE].

[21] L.F. Alday, Large Spin Perturbation Theory, arXiv:1611.01500 [INSPIRE].

[22] R. Gopakumar, A. Kaviraj, K. Sen and A. Sinha, Conformal Bootstrap in Mellin Space, Phys. Rev. Lett. 118 (2017) 081601 [arXiv: 1609. 00572] [INSPIRE].

[23] A. Guerrieri, A.C. Petkou and C. Wen, The free $\sigma$ CFTs, JHEP 09 (2016) 019 [arXiv: 1604.07310] [INSPIRE].

[24] J.M. Maldacena, The Large- $N$ limit of superconformal field theories and supergravity, Int. J. Theor. Phys. 38 (1999) 1113 [hep-th/9711200] [InSPIRE].

[25] S.S. Gubser, I.R. Klebanov and A.M. Polyakov, Gauge theory correlators from noncritical string theory, Phys. Lett. B 428 (1998) 105 [hep-th/9802109] [INSPIRE].

[26] E. Witten, Anti-de Sitter space and holography, Adv. Theor. Math. Phys. 2 (1998) 253 [hep-th/9802150] [INSPIRE]. 
[27] I. Heemskerk, J. Penedones, J. Polchinski and J. Sully, Holography from Conformal Field Theory, JHEP 10 (2009) 079 [arXiv:0907.0151] [INSPIRE].

[28] S. El-Showk and K. Papadodimas, Emergent Spacetime and Holographic CFTs, JHEP 10 (2012) 106 [arXiv: 1101.4163] [INSPIRE].

[29] M. Gary, S.B. Giddings and J. Penedones, Local bulk S-matrix elements and CFT singularities, Phys. Rev. D 80 (2009) 085005 [arXiv:0903.4437] [InSPIRE].

[30] A.L. Fitzpatrick and J. Kaplan, AdS Field Theory from Conformal Field Theory, JHEP 02 (2013) 054 [arXiv: 1208.0337] [inSPIRE].

[31] X. Bekaert, J. Erdmenger, D. Ponomarev and C. Sleight, Quartic AdS Interactions in Higher-Spin Gravity from Conformal Field Theory, JHEP 11 (2015) 149 [arXiv:1508.04292] [INSPIRE].

[32] E.D. Skvortsov and M. Taronna, On Locality, Holography and Unfolding, JHEP 11 (2015) 044 [arXiv: 1508.04764] [INSPIRE].

[33] J. Maldacena, D. Simmons-Duffin and A. Zhiboedov, Looking for a bulk point, JHEP 01 (2017) 013 [arXiv: 1509.03612] [inSPIRE].

[34] M. Taronna, Pseudo-local Theories: A Functional Class Proposal, in proceedings of the International Workshop on Higher Spin Gauge Theories, Singapore, Singapore, November 4-6, 2015 [arXiv: 1602.08566] [INSPIRE].

[35] X. Bekaert, J. Erdmenger, D. Ponomarev and C. Sleight, Bulk quartic vertices from boundary four-point correlators, in proceedings of the International Workshop on Higher Spin Gauge Theories, Singapore, Singapore, November 4-6, 2015 [arXiv:1602.08570] [INSPIRE].

[36] M. Taronna, A note on field redefinitions and higher-spin equations, J. Phys. A 50 (2017) 075401 [arXiv : 1607.04718] [INSPIRE].

[37] C. Sleight, Interactions in Higher-Spin Gravity: a Holographic Perspective, Ph.D. Thesis, University of Munich, Munich Germany (2016), arXiv:1610.01318 [INSPIRE].

[38] A. Belin, B. Freivogel, R.A. Jefferson and L. Kabir, Sub-AdS scale locality in $A d S_{3} / C F T_{2}$, JHEP 04 (2017) 147 [arXiv:1611.08601] [INSPIRE].

[39] G. Barnich and M. Henneaux, Consistent couplings between fields with a gauge freedom and deformations of the master equation, Phys. Lett. B 311 (1993) 123 [hep-th/9304057] [INSPIRE].

[40] M.S. Costa, V. Gonçalves and J. Penedones, Spinning AdS Propagators, JHEP 09 (2014) 064 [arXiv: 1404.5625] [INSPIRE].

[41] S. Ferrara and G. Parisi, Conformal covariant correlation functions, Nucl. Phys. B 42 (1972) 281 [INSPIRE].

[42] S. Ferrara, A.F. Grillo and G. Parisi, Nonequivalence between conformal covariant wilson expansion in euclidean and minkowski space, Lett. Nuovo Cim. 5 (1972) 147.

[43] C. Fronsdal, Elementary particles in a curved space. II, Phys. Rev. D 10 (1974) 589 [INSPIRE].

[44] A.C. Petkou, Evaluating the AdS dual of the critical $O(N)$ vector model, JHEP 03 (2003) 049 [hep-th/0302063] [INSPIRE]. 
[45] X. Bekaert, J. Erdmenger, D. Ponomarev and C. Sleight, Towards holographic higher-spin interactions: Four-point functions and higher-spin exchange, JHEP 03 (2015) 170 [arXiv:1412.0016] [INSPIRE].

[46] E.D. Skvortsov, On (Un)Broken Higher-Spin Symmetry in Vector Models, arXiv: 1512.05994 [INSPIRE].

[47] C. Sleight and M. Taronna, Higher Spin Interactions from Conformal Field Theory: The Complete Cubic Couplings, Phys. Rev. Lett. 116 (2016) 181602 [arXiv:1603.00022] [INSPIRE].

[48] D.J. Gross and V. Rosenhaus, The Bulk Dual of SYK: Cubic Couplings, JHEP 05 (2017) 092 [arXiv: 1702.08016] [INSPIRE].

[49] S.S. Gubser and S. Parikh, Geodesic bulk diagrams on the Bruhat-Tits tree, arXiv: 1704.01149 [INSPIRE].

[50] R. Gopakumar, A. Kaviraj, K. Sen and A. Sinha, A Mellin space approach to the conformal bootstrap, JHEP 05 (2017) 027 [arXiv:1611.08407] [INSPIRE].

[51] P. Dey, A. Kaviraj and A. Sinha, Mellin space bootstrap for global symmetry, arXiv: 1612.05032 [INSPIRE].

[52] O. Aharony, L.F. Alday, A. Bissi and E. Perlmutter, Loops in AdS from Conformal Field Theory, arXiv:1612.03891 [INSPIRE].

[53] C. Sleight and M. Taronna, Higher-Spin Algebras, Holography and Flat Space, JHEP 02 (2017) 095 [arXiv: 1609.00991] [INSPIRE].

[54] E. Hijano, P. Kraus, E. Perlmutter and R. Snively, Witten Diagrams Revisited: The AdS Geometry of Conformal Blocks, JHEP 01 (2016) 146 [arXiv:1508.00501] [INSPIRE].

[55] T. Hartman, Entanglement Entropy at Large Central Charge, arXiv:1303.6955 [INSPIRE].

[56] A.L. Fitzpatrick, J. Kaplan and M.T. Walters, Universality of Long-Distance AdS Physics from the CFT Bootstrap, JHEP 08 (2014) 145 [arXiv: 1403.6829] [INSPIRE].

[57] C.T. Asplund, A. Bernamonti, F. Galli and T. Hartman, Holographic Entanglement Entropy from 2d CFT: Heavy States and Local Quenches, JHEP 02 (2015) 171 [arXiv: 1410.1392] [INSPIRE].

[58] J. de Boer, A. Castro, E. Hijano, J.I. Jottar and P. Kraus, Higher spin entanglement and $\mathcal{W}_{\mathrm{N}}$ conformal blocks, JHEP 07 (2015) 168 [arXiv:1412.7520] [INSPIRE].

[59] E. Hijano, P. Kraus and R. Snively, Worldline approach to semi-classical conformal blocks, JHEP 07 (2015) 131 [arXiv: 1501.02260] [INSPIRE].

[60] A.L. Fitzpatrick, J. Kaplan and M.T. Walters, Virasoro Conformal Blocks and Thermality from Classical Background Fields, JHEP 11 (2015) 200 [arXiv:1501.05315] [INSPIRE].

[61] K.B. Alkalaev and V.A. Belavin, Classical conformal blocks via AdS/CFT correspondence, JHEP 08 (2015) 049 [arXiv: 1504.05943] [INSPIRE].

[62] E. Hijano, P. Kraus, E. Perlmutter and R. Snively, Semiclassical Virasoro blocks from AdS $S_{3}$ gravity, JHEP 12 (2015) 077 [arXiv: 1508.04987] [INSPIRE].

[63] M. Nishida and K. Tamaoka, Geodesic Witten diagrams with an external spinning field, arXiv: 1609. 04563 [INSPIRE]. 
[64] A. Castro, E. Llabrés and F. Rejon-Barrera, Geodesic Diagrams, Gravitational Interactions Eamp; OPE Structures, arXiv:1702.06128 [INSPIRE].

[65] E. Dyer, D.Z. Freedman and J. Sully, Spinning Geodesic Witten Diagrams, arXiv: 1702.06139 [INSPIRE].

[66] H.-Y. Chen, E.-J. Kuo and H. Kyono, Anatomy of Geodesic Witten Diagrams, JHEP 05 (2017) 070 [arXiv: 1702.08818] [InSPIRE].

[67] D. Poland and D. Simmons-Duffin, Bounds on 4D Conformal and Superconformal Field Theories, JHEP 05 (2011) 017 [arXiv: 1009.2087] [INSPIRE].

[68] D. Poland, D. Simmons-Duffin and A. Vichi, Carving Out the Space of 4 D CFTs, JHEP 05 (2012) 110 [arXiv:1109.5176] [INSPIRE].

[69] M.S. Costa, J. Penedones, D. Poland and S. Rychkov, Spinning Conformal Blocks, JHEP 11 (2011) 154 [arXiv:1109.6321] [INSPIRE].

[70] A. Castedo Echeverri, E. Elkhidir, D. Karateev and M. Serone, Deconstructing Conformal Blocks in 4D CFT, JHEP 08 (2015) 101 [arXiv:1505.03750] [INSPIRE].

[71] L. Iliesiu, F. Kos, D. Poland, S.S. Pufu, D. Simmons-Duffin and R. Yacoby, Bootstrapping $3 D$ Fermions, JHEP 03 (2016) 120 [arXiv:1508.00012] [INSPIRE].

[72] A. Castedo Echeverri, E. Elkhidir, D. Karateev and M. Serone, Seed Conformal Blocks in $4 D$ CFT, JHEP 02 (2016) 183 [arXiv:1601.05325] [INSPIRE].

[73] M. Hogervorst, Dimensional Reduction for Conformal Blocks, JHEP 09 (2016) 017 [arXiv: 1604.08913] [INSPIRE].

[74] M. Hogervorst and S. Rychkov, Radial Coordinates for Conformal Blocks, Phys. Rev. D 87 (2013) 106004 [arXiv:1303.1111] [InSPIRE].

[75] M. Hogervorst, H. Osborn and S. Rychkov, Diagonal Limit for Conformal Blocks in d Dimensions, JHEP 08 (2013) 014 [arXiv:1305.1321] [INSPIRE].

[76] M.S. Costa, T. Hansen, J. Penedones and E. Trevisani, Radial expansion for spinning conformal blocks, JHEP 07 (2016) 057 [arXiv: 1603.05552] [INSPIRE].

[77] L. Hoffmann, A.C. Petkou and W. Rühl, A Note on the analyticity of AdS scalar exchange graphs in the crossed channel, Phys. Lett. B 478 (2000) 320 [hep-th/0002025] [INSPIRE].

[78] L. Hoffmann, A.C. Petkou and W. Rühl, Aspects of the conformal operator product expansion in AdS/CFT correspondence, Adv. Theor. Math. Phys. 4 (2002) 571 [hep-th/0002154] [INSPIRE].

[79] D. Simmons-Duffin, Projectors, Shadows and Conformal Blocks, JHEP 04 (2014) 146 [arXiv:1204.3894] [INSPIRE].

[80] M.S. Costa and T. Hansen, Conformal correlators of mixed-symmetry tensors, JHEP 02 (2015) 151 [arXiv:1411.7351] [InSPIRE].

[81] F. Rejon-Barrera and D. Robbins, Scalar-Vector Bootstrap, JHEP 01 (2016) 139 [arXiv: 1508. 02676] [INSPIRE].

[82] T.Y. Thomas, On conformal geometry, in proceedings of the National Academy of Sciences of the United States of America 12 (1926) 352. 
[83] V.K. Dobrev, V.B. Petkova, S.G. Petrova and I.T. Todorov, Dynamical Derivation of Vacuum Operator Product Expansion in Euclidean Conformal Quantum Field Theory, Phys. Rev. D 13 (1976) 887 [InSPIRE].

[84] V.K. Dobrev, G. Mack, V.B. Petkova, S.G. Petrova and I.T. Todorov, Harmonic Analysis: on the n-Dimensional Lorentz Group and Its Application to Conformal Quantum Field Theory, in Lecture Notes in Physics 63 (1977), Springer-Verlag.

[85] R.R. Metsaev, Cubic interaction vertices of massive and massless higher spin fields, Nucl. Phys. B 759 (2006) 147 [hep-th/0512342] [INSPIRE].

[86] M.S. Costa, J. Penedones, D. Poland and S. Rychkov, Spinning Conformal Correlators, JHEP 11 (2011) 071 [arXiv:1107.3554] [INSPIRE].

[87] H. Osborn and A.C. Petkou, Implications of conformal invariance in field theories for general dimensions, Annals Phys. 231 (1994) 311 [hep-th/9307010] [INSPIRE].

[88] J. Erdmenger and H. Osborn, Conserved currents and the energy momentum tensor in conformally invariant theories for general dimensions, Nucl. Phys. B 483 (1997) 431 [hep-th/9605009] [INSPIRE].

[89] Y.S. Stanev, Correlation Functions of Conserved Currents in Four Dimensional Conformal Field Theory, Nucl. Phys. B 865 (2012) 200 [arXiv:1206.5639] [InSPIRE].

[90] A. Zhiboedov, A note on three-point functions of conserved currents, arXiv:1206.6370 [INSPIRE].

[91] J. Maldacena and A. Zhiboedov, Constraining Conformal Field Theories with A Higher Spin Symmetry, J. Phys. A 46 (2013) 214011 [arXiv:1112.1016] [inSPIRE].

[92] N. Boulanger, D. Ponomarev, E.D. Skvortsov and M. Taronna, On the uniqueness of higher-spin symmetries in AdS and CFT, Int. J. Mod. Phys. A 28 (2013) 1350162 [arXiv: 1305.5180] [INSPIRE].

[93] V. Alba and K. Diab, Constraining conformal field theories with a higher spin symmetry in $D=4$, arXiv: 1307.8092 [INSPIRE].

[94] V. Alba and K. Diab, Constraining conformal field theories with a higher spin symmetry in $d>3$ dimensions, JHEP 03 (2016) 044 [arXiv: 1510.02535] [INSPIRE].

[95] D. Friedan and C.A. Keller, Cauchy Conformal Fields in Dimensions d $>2$, Commun. Math. Phys. 348 (2016) 655 [arXiv: 1509.07475] [INSPIRE].

[96] D. Francia, J. Mourad and A. Sagnotti, Current Exchanges and Unconstrained Higher Spins, Nucl. Phys. B 773 (2007) 203 [hep-th/0701163] [inSPIRE].

[97] D. Francia, J. Mourad and A. Sagnotti, $(A) d S$ exchanges and partially-massless higher spins, Nucl. Phys. B 804 (2008) 383 [arXiv:0803.3832] [InSPIRE].

[98] J. Penedones, High Energy Scattering in the AdS/CFT Correspondence, arXiv:0712.0802 [INSPIRE].

[99] M. Taronna, Higher-Spin Interactions: three-point functions and beyond, arXiv:1209.5755 [INSPIRE].

[100] D.Z. Freedman, S.D. Mathur, A. Matusis and L. Rastelli, Correlation functions in the CFT $(d) / A d S(d+1)$ correspondence, Nucl. Phys. B 546 (1999) 96 [hep-th/9804058] [INSPIRE].

[101] A. Mikhailov, Notes on higher spin symmetries, hep-th/0201019 [INSPIRE]. 
[102] B. Allen, The Graviton Propagator in Homogeneous and Isotropic Space-times, Nucl. Phys. B 287 (1987) 743 [INSPIRE].

[103] B. Allen and T. Jacobson, Vector Two Point Functions in Maximally Symmetric Spaces, Commun. Math. Phys. 103 (1986) 669 [INSPIRE].

[104] M. Turyn, The Graviton Propagator in Maximally Symmetric Spaces, J. Math. Phys. 31 (1990) 669 [INSPIRE].

[105] H. Liu and A.A. Tseytlin, On four point functions in the CFT/AdS correspondence, Phys. Rev. D 59 (1999) 086002 [hep-th/9807097] [INSPIRE].

[106] E. D'Hoker and D.Z. Freedman, Gauge boson exchange in AdS(d+1), Nucl. Phys. B 544 (1999) 612 [hep-th/9809179] [INSPIRE].

[107] E. D'Hoker, D.Z. Freedman, S.D. Mathur, A. Matusis and L. Rastelli, Graviton and gauge boson propagators in AdS(d+1), Nucl. Phys. B 562 (1999) 330 [hep-th/9902042] [INSPIRE].

[108] C. Fronsdal, Singletons and Massless, Integral Spin Fields on de Sitter Space, Phys. Rev. D 20 (1979) 848 [INSPIRE].

[109] T. Leonhardt, R. Manvelyan and W. Rühl, The Group approach to AdS space propagators, Nucl. Phys. B 667 (2003) 413 [hep-th/0305235] [INSPIRE].

[110] T. Leonhardt, W. Rühl and R. Manvelyan, The Group approach to AdS space propagators: A Fast algorithm, J. Phys. A 37 (2004) 7051 [hep-th/0310063] [INSPIRE].

[111] R. Manvelyan and W. Rühl, The Off-shell behaviour of propagators and the Goldstone field in higher spin gauge theory on AdS(d+1) space, Nucl. Phys. B 717 (2005) 3 [hep-th/0502123] [INSPIRE].

[112] R. Manvelyan, K. Mkrtchyan and W. Rühl, Ultraviolet behaviour of higher spin gauge field propagators and one loop mass renormalization, Nucl. Phys. B 803 (2008) 405 [arXiv:0804.1211] [INSPIRE].

[113] K. Mkrtchyan, Higher Spin Interacting Quantum Field Theory and Higher Order Conformal Invariant Lagrangians, Ph.D. Thesis, Yerevan Physics Institute, Yerevan, Armenia, arXiv: 1011.0160 [INSPIRE].

[114] L.P.S. Singh and C.R. Hagen, Lagrangian formulation for arbitrary spin. 1. The boson case, Phys. Rev. D 9 (1974) 898 [inSPIRE].

[115] L.P.S. Singh and C.R. Hagen, Lagrangian formulation for arbitrary spin. 2. The fermion case, Phys. Rev. D 9 (1974) 910 [inSPIRE].

[116] S.D. Rindani and M. Sivakumar, Gauge-invariant description of massive higher-spin particles by dimensional reduction, Phys. Rev. D 32 (1985) 3238 [InSPIRE].

[117] S.D. Rindani, D. Sahdev and M. Sivakumar, Dimensional reduction of symmetric higher spin actions. 1. Bosons, Mod. Phys. Lett. A 4 (1989) 265 [inSPIRE].

[118] C. Aragone, S. Deser and Z. Yang, Massive Higher Spin From Dimensional Reduction of Gauge Fields, Annals Phys. 179 (1987) 76 [inSPIRE].

[119] Yu. M. Zinoviev, On massive high spin particles in AdS, hep-th/0108192 [INSPIRE].

[120] P.A.M. Dirac, Relativistic wave equations, Proc. Roy. Soc. Lond. A 155 (1936) 447 [INSPIRE].

[121] M. Fierz, Force-free particles with any spin, Helv. Phys. Acta 12 (1939) 3 [INSPIRE]. 
[122] M. Fierz and W. Pauli, On relativistic wave equations for particles of arbitrary spin in an electromagnetic field, Proc. Roy. Soc. Lond. A 173 (1939) 211.

[123] D. Francia, Geometric Lagrangians for massive higher-spin fields, Nucl. Phys. B 796 (2008) 77 [arXiv: 0710.5378] [INSPIRE].

[124] D. Francia, Geometric massive higher spins and current exchanges, Fortsch. Phys. 56 (2008) 800 [arXiv: 0804.2857] [inSPIRE].

[125] C. Fronsdal, Massless Fields with Integer Spin, Phys. Rev. D 18 (1978) 3624 [InSPIRE].

[126] D. Francia and A. Sagnotti, Free geometric equations for higher spins, Phys. Lett. B 543 (2002) 303 [hep-th/0207002] [INSPIRE].

[127] D. Francia and A. Sagnotti, Minimal local Lagrangians for higher-spin geometry, Phys. Lett. B 624 (2005) 93 [hep-th/0507144] [INSPIRE].

[128] D. Francia, On the Relation between Local and Geometric Lagrangians for Higher spins, J. Phys. Conf. Ser. 222 (2010) 012002 [arXiv:1001.3854] [INSPIRE].

[129] H. Liu, Scattering in anti-de Sitter space and operator product expansion, Phys. Rev. D 60 (1999) 106005 [hep-th/9811152] [INSPIRE].

[130] D.Z. Freedman, S.D. Mathur, A. Matusis and L. Rastelli, Comments on 4 point functions in the CFT/AdS correspondence, Phys. Lett. B 452 (1999) 61 [hep-th/9808006] [INSPIRE].

[131] E. D'Hoker and D.Z. Freedman, General scalar exchange in AdS(d+1), Nucl. Phys. B 550 (1999) 261 [hep-th/9811257] [INSPIRE].

[132] E. D'Hoker, D.Z. Freedman, S.D. Mathur, A. Matusis and L. Rastelli, Graviton exchange and complete four point functions in the AdS/CFT correspondence, Nucl. Phys. B 562 (1999) 353 [hep-th/9903196] [INSPIRE].

[133] G. Arutyunov and S. Frolov, Four point functions of lowest weight CPOs in $N=4 S Y M(4)$ in supergravity approximation, Phys. Rev. D 62 (2000) 064016 [hep-th/0002170] [INSPIRE].

[134] G. Arutyunov, S. Frolov and A.C. Petkou, Operator product expansion of the lowest weight CPOs in $\mathcal{N}=4 S_{Y M}$ at strong coupling, Nucl. Phys. B 586 (2000) 547 [Erratum ibid. B 609 (2001) 539] [hep-th/0005182] [INSPIRE].

[135] L. Hoffmann, L. Mesref and W. Rühl, AdS box graphs, unitarity and operator product expansions, Nucl. Phys. B 589 (2000) 337 [hep-th/0006165] [InSPIRE].

[136] G. Arutyunov, F.A. Dolan, H. Osborn and E. Sokatchev, Correlation functions and massive Kaluza-Klein modes in the AdS/CFT correspondence, Nucl. Phys. B 665 (2003) 273 [hep-th/0212116] [INSPIRE].

[137] L.I. Uruchurtu, AdS/CFT for Four-Point Amplitudes involving Gravitino Exchange, JHEP 09 (2007) 086 [arXiv:0707.0424] [INSPIRE].

[138] P. Haggi-Mani and B. Sundborg, Free large- $N$ supersymmetric Yang-Mills theory as a string theory, JHEP 04 (2000) 031 [hep-th/0002189] [INSPIRE].

[139] B. Sundborg, Stringy gravity, interacting tensionless strings and massless higher spins, Nucl. Phys. Proc. Suppl. 102 (2001) 113 [hep-th/0103247] [INSPIRE].

[140] E. Witten, Spacetime Reconstruction, talk given at the J.H. Schwarz 60-th Birthday Conference, California Insitute of Technology, Pasadena, U.S.A. November 3-4, 2001, http://theory.caltech.edu/jhs60/witten/1.html. 
[141] E. Sezgin and P. Sundell, Massless higher spins and holography, Nucl. Phys. B 644 (2002) 303 [Erratum ibid. B 660 (2003) 403] [hep-th/0205131] [INSPIRE].

[142] I.R. Klebanov and A.M. Polyakov, AdS dual of the critical $O(N)$ vector model, Phys. Lett. B 550 (2002) 213 [hep-th/0210114] [INSPIRE].

[143] R.G. Leigh and A.C. Petkou, Holography of the $N=1$ higher spin theory on AdS $S_{4}$, JHEP 06 (2003) 011 [hep-th/0304217] [INSPIRE].

[144] M.A. Vasiliev, Nonlinear equations for symmetric massless higher spin fields in (A)dS(d), Phys. Lett. B 567 (2003) 139 [hep-th/0304049] [INSPIRE].

[145] S. Giombi and X. Yin, Higher Spin Gauge Theory and Holography: The Three-Point Functions, JHEP 09 (2010) 115 [arXiv:0912.3462] [INSPIRE].

[146] S. Giombi and X. Yin, Higher Spins in AdS and Twistorial Holography, JHEP 04 (2011) 086 [arXiv: 1004.3736] [INSPIRE].

[147] C.-M. Chang and X. Yin, Higher Spin Gravity with Matter in AdS $S_{3}$ and Its CFT Dual, JHEP 10 (2012) 024 [arXiv:1106.2580] [inSPIRE].

[148] M. Ammon, P. Kraus and E. Perlmutter, Scalar fields and three-point functions in D $=3$ higher spin gravity, JHEP 07 (2012) 113 [arXiv:1111.3926] [INSPIRE].

[149] E. Hijano, P. Kraus and E. Perlmutter, Matching four-point functions in higher spin $A d S_{3} / C F T_{2}, J H E P 05$ (2013) 163 [arXiv: 1302.6113] [INSPIRE].

[150] E. Joung and M. Taronna, Cubic interactions of massless higher spins in (A)dS: metric-like approach, Nucl. Phys. B 861 (2012) 145 [arXiv:1110.5918] [INSPIRE].

[151] E. Joung, L. Lopez and M. Taronna, Solving the Noether procedure for cubic interactions of higher spins in (A)dS, J. Phys. A 46 (2013) 214020 [arXiv:1207.5520] [InSPIRE].

[152] E. Joung, L. Lopez and M. Taronna, Generating functions of (partially-)massless higher-spin cubic interactions, JHEP 01 (2013) 168 [arXiv:1211.5912] [INSPIRE].

[153] E. Joung and M. Taronna, Cubic-interaction-induced deformations of higher-spin symmetries, JHEP 03 (2014) 103 [arXiv:1311.0242] [INSPIRE].

[154] M. Grigoriev and A. Waldron, Massive Higher Spins from BRST and Tractors, Nucl. Phys. B 853 (2011) 291 [arXiv:1104.4994] [INSPIRE].

[155] C. Sleight, Lectures on Higher Spin Holography, arXiv:1701.08360 [INSPIRE].

[156] J. Penedones, Writing CFT correlation functions as AdS scattering amplitudes, JHEP 03 (2011) 025 [arXiv: 1011.1485] [INSPIRE].

[157] M.F. Paulos, Towards Feynman rules for Mellin amplitudes, JHEP 10 (2011) 074 [arXiv:1107.1504] [INSPIRE]. 